\title{
Directional Thermodynamic Formalism
}

\author{
Mourad Ben Slimane ${ }^{1, *}$, Moez Ben Abid ${ }^{2}$, Ines Ben Omrane ${ }^{3}$ and Borhen Halouani ${ }^{1}$ \\ 1 Department of Mathematics, College of Science, King Saud University, P.O. Box 2455, \\ Riyadh 11451, Saudi Arabia; halouani@ksu.edu.sa \\ 2 Ecole Supérieure des Sciences et de la Technologie de Hammam Sousse, Université de Sousse, \\ Sousse 4011, Tunisia; moezbenabid@yahoo.fr \\ 3 Department of Mathematics, Faculty of Science, Imam Mohammad Ibn Saud Islamic University (IMSIU), \\ P.O. Box 90950, Riyadh 11623, Saudi Arabia; imbenomrane@imamu.edu.sa \\ * Correspondence: mbenslimane@ksu.edu.sa
}

Received: 5 April 2019; Accepted: 4 June 2019; Published: 21 June 2019

\begin{abstract}
The usual thermodynamic formalism is uniform in all directions and, therefore, it is not adapted to study multi-dimensional functions with various directional behaviors. It is based on a scaling function characterized in terms of isotropic Sobolev or Besov-type norms. The purpose of the present paper was twofold. Firstly, we proved wavelet criteria for a natural extended directional scaling function expressed in terms of directional Sobolev or Besov spaces. Secondly, we performed the directional multifractal formalism, i.e., we computed or estimated directional Hölder spectra, either directly or via some Legendre transforms on either directional scaling function or anisotropic scaling functions. We obtained general upper bounds for directional Hölder spectra. We also showed optimal results for two large classes of examples of deterministic and random anisotropic self-similar tools for possible modeling turbulence (or cascades) and textures in images: Sierpinski cascade functions and fractional Brownian sheets.
\end{abstract}

Keywords: directional hölder regularity; anisotropic hölder regularity; directional scaling function; anisotropic scaling function; directional multifractal formalism; wavelet bases; sierpinski cascade functions; fractional brownian sheets

MSC: 26A15; 26A16; 26B35; 26B05; 46E35; 46E99; 42C40

\section{Introduction}

Multifractal models were originally proposed to describe the intermittent behavior of fully-developed turbulence [1,2] and also chaotic features in dynamical systems [3,4]. If $T$ is the time and $v(T)$ is a the stream-wise component of the velocity of a turbulent flow at a given point, Kolmogorov [5] expected a power law behavior:

$$
\int_{\mathbb{R}}|v(T+t)-v(T)|^{p} d T \sim|t|^{\eta_{L}(p)} \quad \text { for small }|t|,
$$

with $\eta_{L}(p)=p / 3$. Kolmogorov and Oboukhov and [6,7] have refined this prediction into a quadratic behavior.

Various experimental results and other models have confirm the nonlinear behavior. Mandelbrot $[2,8,9]$ has introduced multiplicative cascades for the dissipation of energy in turbulent flows and thus has associated fractals to measures (or functions). Frisch and Parisi [10] conjectured that $\eta_{L}(p)$ describes the statistical repartition of the pointwise Lipschitz regularities. The Lipschitz 
spectrum of $v$ is the map which associates to each $0<H<1$ the Hausdorff dimension $d(H)$ of the set of times $T$ where $v$ has a given pointwise Lipschitz regularity $h_{v}(T)=H$ in the sense that:

$$
|v(T+t)-v(T)| \sim|t|^{H} \quad \text { for small }|t| .
$$

By heuristic arguments, the thermodynamic formalism [10] states that $d(H)$ is given by the following Legendre transform of $\eta_{L}(p)$ :

$$
d(H)=\inf _{p}\left(H p-\eta_{L}(p)+1\right) .
$$

A similar formalism for measures has been derived; if $\mu$ is a probability measure on $\mathbb{R}^{d}$, the notion of pointwise Lipschitz regularity is replaced by the local regularity of $\mu$ defined as:

$$
h_{\mu}(x)=\liminf _{r \rightarrow 0} \frac{\log \mu(B(x, r))}{\log r},
$$

(see $[3,4,11-20] \ldots$.$) . Note that if d=1$ and $h_{\mu}(T) \in[0,1)$, then $h_{f}(T)=h_{\mu}(T)$ for $f(x)=\mu((-\infty, x])$.

In [21], Daubechies and Lagarias proved the validity of the thermodynamic formalism (3) for some refinement functions used in the construction of orthonormal wavelet bases in one dimension.

An alternative formulation of the Lipschitz scaling function $\eta_{L}(p)$ in terms of continuous wavelet transform $C_{a, b}=\frac{1}{a^{d}} \int_{\mathbb{R}^{d}} f(x) \psi\left(\frac{x-b}{a}\right) d x\left(a>0\right.$ and $\left.b \in \mathbb{R}^{d}\right)$ was proposed by Arneodo, Bacry and Muzy in $\mathbb{R}^{d}$ with $d=1$ (see [22-24]). The corresponding thermodynamic formalism was proved in [22] for the primitive of a multinomial measure or a $C^{1}$ perturbation of such a measure.

Jaffard [25] extended these formulae in $\mathbb{R}^{d}$ and showed the link between them via the function space interpretation of the scaling function in terms of (isotropic) Sobolev or Besov-type norms. Such spaces for smoothness index higher than 1 are also characterized by finite order differences. The corresponding scaling function $\eta(p)$ (given in (14)) is expected to give information for the Hölder spectrum $d(H)$ for any value of $H$. The scaling function $\eta(p)$ is also characterized by either isotropic wavelet bases (i.e., decompositions on tensor products of one-dimensional wavelets with the same dilation factor $2^{j}$ at scale $j$ in all coordinate axes) or continuous wavelet transform (in fact, $\left.\eta(p)=\liminf _{a \rightarrow 0} \frac{\log \int\left|C_{a, b}\right|^{p} d b}{\log a}\right)$.

The scope of the mathematical validity of the thermodynamic formalism,

$$
d(H)=\inf _{p}(H p-\eta(p)+d)
$$

has become an important issue. The general rule for a good multifractal formalism is to get optimal upper bounds for the spectra. Optimality is obtained for examples that saturate the upper bound, i.e., the upper bound becomes equality.

If the range $P$ of $p$ 's over which one computes the Legendre transform is chosen appropriately:

$$
P=\{p: \quad \eta(p)>d\},
$$

then (5) yields an upper bound for all functions [25].

The optimality has been either studied or proved under self-similarity assumptions [21,22,25-31], or for a class of particular random processes [32], or for specific functions [33,34], or even generically in either Baire's categories [35] or prevalence sense [36,37].

Alternatively, Kestener and Arneodo (see [38] and references therein) proposed, also for $d=2$ and $d=3$, different vectoriel wavelet transform formulas. They applied them to turbulent velocity and vorticity $3 \mathrm{D}$ numerical data. 
Unfortunately, for $d \geq 2$, the scaling functions $\eta_{L}(p)$ and $\eta(p)$ are uniform in all directions, and, therefore, are not adapted to study images and multi-dimensional signals with various directional behaviors. These behaviors are important for detection of edges, efficient image compression, texture classification, etc., (see, for instance, [39-51] and the references therein). They also appear in partial differential equations, pseudo-differential operators theory, approximation theory, etc. (for example, see [52] or [53] and references therein). In that case, signals present anisotropies quantified through regularity characteristics and features that strongly differ when measured in different directions [17,19,46,47,49,54-56]. Classes of functions that satisfy different scaling properties according the coordinate axes have been studied in $[29,42,44,46,49-51,53-55,57-72]$. For an a priori prescribed anisotropy $\alpha=\left(\alpha_{1}, \cdots, \alpha_{d}\right)$ with $0<\alpha_{1}, \cdots, \alpha_{d}$ and $\sum_{i=1}^{d} \alpha_{i}=d$, such signals can be expanded in Triebel bases (i.e., tensor products of one-dimensional wavelets that allow dilations factors about $2^{j \alpha_{1}}, \ldots, 2^{j \alpha_{d}}$ in coordinate axes [69,70]). Alternatively, we can also use anisotropic continuous wavelet transform $\frac{1}{a^{d}} \int_{\mathbb{R}^{d}} f(x) \psi\left(\frac{x_{1}-b_{1}}{a^{\alpha_{1}}}, \ldots, \frac{x_{d}-b_{d}}{a^{\alpha_{d}}}\right) d x$ (see [45,48]). Signals where no a priori anisotropy is prescribed, can be expanded in DeVore, Konyagin, and Temlyakov hyperbolic wavelet bases [73], i.e., tensor products of one-dimensional wavelets that allow different dilations factors $2^{j_{1}}, \ldots, 2^{j_{d}}$ in coordinate axes (see also [39,74-76]. Hyperbolic wavelet bases contain all possible anisotropies.

Both Triebel and hyperbolic bases characterize anisotropic Besov spaces [39,70].

Let $d \geq 2$ be a positive integer. Let $e$ be a fixed vector in the unit sphere $S^{d-1}$. We will focus on the following global and local directional behaviors:

- A natural directional Lipschitz scaling function $\eta_{L}(p, e)$ of $v$ in direction $e$ can be given by:

$$
\int_{\mathbb{R}^{d}}|v(y+t e)-v(y)|^{p} d y \sim|t|^{\eta_{L}(p, e)} \quad \text { for small }|t| .
$$

It can be extended to a directional scaling function $\eta(p, e)$ that involves any finite order differences in direction $e$ (see Definition 1). It can be also restricted to a bounded domain (see Definition 3).

- A natural directional pointwise Lipschitz regularity $h_{L}(y, e)$ of $v$ at $y$ in direction $e$ can be given by:

$$
|v(y+t e)-v(y)| \sim|t|^{h_{L}(y, e)} \quad \text { for small }|t| .
$$

It can be extended to a directional pointwise Hölder regularity $h(y, e)$ (see Definition 5).

In this paper, we want to understand how singularities given by directional pointwise Hölder regularities fluctuate from point to point for a fixed direction $e$. These singularities may share a given value on a fractal set. One wishes to compute the Hausdorff dimension of this set. One also wishes to derive this size from global quantities extracted from the signal, given by either a directional scaling function or anisotropic scaling functions. Firstly, we prove wavelet criteria for the directional scaling function $\eta(p, e)$ (see Sections 1-3). Secondly, we perform directional multifractal formalism (see Sections 4-6), i.e., we compute or estimate directional Hölder spectra either directly or via some Legendre transforms on either directional scaling function or anisotropies scaling functions. Two types of results will be performed:

- We will obtain general upper bounds for the directional Hölder spectra.

- We will show optimal results for two large classes of examples of deterministic and random anisotropic self-similar tools for possible modeling turbulence (or cascades) and textures in images (see [50]): Sierpinski cascade functions introduced by the first author in [29] and fractional Brownian sheets introduced by both Kamont in [67] and Pesquet-Popesu and Lévy-Véhel in [77], and revisited by Ayache, Léger, and Pontier [78] for extra properties.

Note that the heuristic classical arguments from which the thermodynamic formalism for pointwise Lipschitz regularity was derived (see, for example, [25], pp. 947-948) cannot be applied to the directional pointwise Lipschitz regularity; near a point $y$ such that $h(y)=H$, we have 
$|v(y+t)-v(y)| \sim|t|^{H}$ in a cube of radius $|t|$. There are about $|t|^{-d(H)}$ such cubes, so that the total contribution to $\int_{\mathbb{R}^{d}}|v(y+t)-v(y)|^{p} d y$ is $|t|^{H p+d-d(H)}$. It follows that $\eta_{L}(p)=\inf _{H}(H p-d(H)+d)$. If $d(H)$ is concave, it is recovered by an inverse Legendre transform formula which yields $d(H)=\inf _{p}\left(H p-\eta_{L}(p)+d\right)$. In the context of directional pointwise Lipschitz regularity, we want to calculate the contribution of critical directional pointwise Lipschitz regularity of order $H$ in direction $e$ to the integral $\int_{\mathbb{R}^{d}}|v(y+t e)-v(y)|^{p} d y$ : near a point $y$ such that $h(y, e)=H$, we have $|v(y+t e)-v(y)| \sim|t|^{H}$ in a small segment of length $|t|$. So, we cannot pursue the above heuristic arguments.

To overcome this inconvenience, we will use a criterion of directional pointwise Hölder regularity $h(y, e)$ in terms of rapid decay of highly oriented multi-scaled wavelet coefficients. Actually, $h(y, e)$ is related to anisotropic pointwise Hölder regularities $h_{\alpha}(y)$, for all anisotropies $\alpha$ (see $[43,44]$ ). The anisotropic pointwise regularity $h_{\alpha}(y)$ was already introduced in [29] and characterized with either anisotropic continuous wavelets or in Triebel bases [43,44].

Alternatively, we will use a characterization of directional pointwise Lipschitz regularity obtained in [58] directly (i.e., without passing through anisotropies) in terms of decay conditions for the coefficients of the expansion in the hyperbolic Schauder basis.

Note that partial characterizations for the directional pointwise Hölder regularity have been obtained by Sampo and Sumetkijakan $[51,79,80]$ (respective to Jaffard [48]), when using parabolic basis, i.e., curvelets and Hart-Smith transform (respective to the anisotropic Gabor-wavelet transform).

Note that other directional behaviors have been studied. Donoho [81], Guo, and Labate [82] have used wedgelets and shearlets for the detection of discontinuities along smooth edges. Candes, Donoho [83], and Mallat [84] have used wavelet bases elongated in particular directions (ridgelets and bandelets) to deal with singularities along lines, along hyperplanes, etc. Fell, Führ, and Voigtlaender [85] characterized the wavefront set in terms of rapid continuous wavelet decay, for a large variety of dilation groups. For the shearlet groups single wavelets suffice, whereas similitude and diagonal groups need suitable families of wavelets. Recently, by using the harmonic wavelet, Sun, Leng, and Cattani [86] constructed a new multilevel system in the Fourier domain, which has the circular shape. This new system is more suitable for the distribution of general images in the Fourier domain.

In the next section, we first give the definition of the directional scaling function $\eta(p, e)$ in terms of directional Sobolev or Besov spaces (expressed by finite order differences) to which $v$ belongs. We then make the connection between directional scaling function and anisotropic scaling functions analyzed in anisotropic function spaces (see Theorem 1).

In Section 3, using the characterization of the anisotropic scaling function in Triebel wavelet bases $[69,70]$, we deduce a criterion of directional scaling function in these bases (see Theorem 2). Then, using the characterization of the anisotropic scaling function in hyperbolic wavelet bases [39], we deduce a criterion of directional scaling function in these bases (see Theorem 3). Finally, using a result of Kamont [87], we deduce a criterion of directional Lipschitz scaling function in hyperbolic Schauder bases without passing through anisotropies (see Theorem 4).

In Section 4, we recall the connection between both directional and anisotropic Hölder regularities (see Proposition 9). We first deduce a general upper bound for the directional Hölder spectrum by anisotropic Hölder spectra (i.e., Hausdorff dimension of anisotropic Hölder sets) (see Theorem 5). Note that in [58], we characterized directional pointwise Lipschitz regularity in terms of decay conditions for the coefficients of the expansion of $f$ in the hyperbolic basis of tensor products of Schauder functions, but we do not yet succeed to deduce a general upper bound for the directional Lipschitz spectrum. We will instead recall a result of [59] in which we adapted the notion of Hausdorff dimension to the anisotropy, we used a criterion of [43] for anisotropic pointwise regularity in terms of conditions on Triebel wavelet coefficients and deduced a general upper bound for the adapted anisotropic Hölder spectrum by means a Legendre transform of the anisotropic scaling function. 
Both this upper bound and Theorem 5 yield a general upper bound for the directional spectrum (see Theorem 6).

In Section 5, we apply Theorem 4 for fractional Brownian sheets to show that unlike the Lipschitz scaling function $\eta_{L}(p)$ and the Lipschitz spectum $d(H)$ which are uniform in all directions, the directional scaling function $\eta_{L}(p, e)$ and the directional spectrum $d(H, e)$ are tools that detect directional behaviors (see Theorems 7 and 8 ). We also prove that if the corresponding appropriate range $P$ of $p^{\prime}$ s over which one will computes the Legendre transform is:

$$
P=\left\{p \geq 1: \quad \eta_{L}(p)>1\right\}=\left\{p \geq 1: \eta_{L}\left(p, e_{i}\right)>1 \forall i \in\{1, \cdots, d\}\right\}
$$

then $\inf _{p \in P}\left(H p-\eta_{L}(p, e)+1\right)$ provides a common directional Lipschitz scaling based directional thermodynamic formalism for these examples (see Theorem 8).

In Section 6, we apply Theorem 4 for Sierpinski cascade functions to show that the directional scaling function $\eta_{L}(p, e)$ and the directional spectrum $d(H, e)$ are tools that detect directional behaviors. We also show that contrary to $\eta_{L}(p, e)$, the directional spectrum $d(H, e)$ depends on the geometric disposition of the chosen contractions for each cascade function. We also provide non common directional Lipschitz scaling based directional thermodynamic formalisms for these examples (see Theorems 9 and 11). These formalisms depend on the geometric disposition of contractions for each cascade function. Nevertheless, all obtained formalisms share the same corresponding appropriate range $P$ of $p$ 's over which one will compute the Legendre transform given in (9). Moreover, we show the optimality of Theorem 6 for Sierpinski cascade functions corresponding to a large class of geometric disposition of contractions (see Theorem 12). Finally, we modify the notion of the Hausdorff dimension to provide a new common directional Lipschitz scaling based directional thermodynamic formalism for all Sierpinski cascade functions (see Theorem 13).

Finally Section 7 motivates the anisotropic cascade model on the physics side.

\section{Directional Scaling Function and Its Connection with Anisotropic Scaling Functions}

\subsection{Directional Scaling Function}

For the definitions of Besov spaces stated in this section, we refer the reader to [69]. Let $d \geq 2$ be a positive integer. Let $e$ be a fixed vector in the unit sphere $S^{d-1}$. For $t \in \mathbb{R}$ and $y \in \mathbb{R}^{d}$, define the difference $\Delta_{t, e} f$ in direction $e$ by the standard formula:

$$
\Delta_{t, e} f(y)=f(y+t e)-f(y)
$$

Define the iterated differences in direction $e$ inductively by

$$
\Delta_{t, e}^{1} f=\Delta_{t, e} f \quad \text { and } \quad \Delta_{t, e}^{n+1} f=\Delta_{t, e}^{1}\left(\Delta_{t, e}^{n} f\right) .
$$

Definition 1. Let $0<s<M$ and $M \in \mathbb{N}, 1 \leq p<\infty$ and $f \in L^{p}\left(\mathbb{R}^{d}\right)$.

We say that $f \in B_{p}^{s}\left(\mathbb{R}^{d}, e\right)$ if there exists $C>0$ such that:

$$
\forall 0<t \leq 1 \quad \int_{\mathbb{R}^{d}}\left|\Delta_{t, e}^{M} f(y)\right|^{p} d y \leq C|t|^{s p} .
$$

Define the directional scaling function $\eta(p, e)$ of $f$ in direction e by:

$$
\eta(p, e)=\sup \left\{s p: f \in B_{p}^{s}\left(\mathbb{R}^{d}, e\right)\right\} .
$$


We say that $f$ belongs to the usual isotropic Besov space $B_{p, \infty}^{s}\left(\mathbb{R}^{d}\right)$ (we will ignore $\infty$ and write $B_{p}^{s}\left(\mathbb{R}^{d}\right)$ ) if there exists $C>0$ such that:

$$
\forall e \in S^{d-1} \forall 0<t \leq 1 \quad \int_{\mathbb{R}^{d}}\left|\Delta_{t, e}^{M} f(y)\right|^{p} d y \leq C|t|^{s p} .
$$

Define the scaling function $\eta(p)$ of $f$ by:

$$
\eta(p)=\sup \left\{s p: f \in B_{p}^{s}\left(\mathbb{R}^{d}\right)\right\} .
$$

Anisotropic Besov spaces were introduced for the study of semi-elliptic pseudo-differential operators whose symbols have different degrees of smoothness along different directions (see [52]; see also [53] and references therein, for a recent use of such spaces for optimal regularity results for the heat equation).

Definition 2. Let $1 \leq p<\infty$. Denote by $\mathcal{D}$ the set $\{1, \cdots, d\}$. For $i \in \mathcal{D}$, let $M_{i} \in \mathbb{N}, 0<s_{i}<M_{i}$ and $e_{i}=\left(\delta_{1, i}, \cdots, \delta_{d, i}\right)$ denotes the $i$-th coordinate vector in $\mathbb{R}^{d}$. The so-called classical anisotropic Besov space $B_{p}^{\left(s_{1}, \cdots, s_{d}\right)}\left(\mathbb{R}^{d}\right)$ is defined as

$$
B_{p}^{\left(s_{1}, \cdots, s_{d}\right)}\left(\mathbb{R}^{d}\right)=\bigcap_{i \in \mathcal{D}} B_{p}^{s_{i}}\left(\mathbb{R}^{d}, e_{i}\right)
$$

Remark 1. When $s_{1}, \cdots, s_{d}=s$, the space $B_{p}^{\left(s_{1}, \cdots, s_{d}\right)}\left(\mathbb{R}^{d}\right)$ coincides with $B_{p}^{s}\left(\mathbb{R}^{d}\right)$.

We will be interested in the characterization of $\eta(p, e)$ in terms of decay conditions for a structure function of the coefficients of the expansion of $f$ in either Triebel anisotropic wavelet bases $[69,70]$ (see Section 3), or hyperbolic wavelet bases [68,73-76] (see Section 4). Without any loss of generality, we can assume that $e=e_{1}$, because we can take coordinates on an orthonormal basis $\mathcal{B}$ of $\mathbb{R}^{d}$ that starts with $e$. Using the partial ordering property,

$$
B_{p}^{\left(s_{1}, \cdots, s_{d}\right)}\left(\mathbb{R}^{d}\right) \subset B_{p}^{\left(s_{1}^{\prime}, \cdots, s_{d}^{\prime}\right)}\left(\mathbb{R}^{d}\right) \quad \forall s_{i}^{\prime} \leq s_{i} \quad \forall i \in \mathcal{D},
$$

we introduce the following substitute for $\eta(p, e)$,

$$
\breve{\eta}(p, e)=\sup \left\{s_{1} p: \exists 0<\varepsilon \leq s_{1} \quad f \in B_{p}^{\left(s_{1}, \varepsilon, \cdots, \varepsilon\right)}\left(\mathbb{R}^{d}\right)\right\} .
$$

We obtain the following result.

Proposition 1. 1. If $\eta(p, e)=0$ then $\breve{\eta}(p, e)=0$.

2. We have always:

$$
\breve{\eta}(p, e) \leq \eta(p, e) .
$$

3. If $\eta(p)>0$ then $\breve{\eta}(p, e)=\eta(p, e)$.

Proof. Both first and second points follow directly from Definition 2.

Assume that $\eta(p)>0$, then $f \in B_{p}^{(\delta, \cdots, \delta)}\left(\mathbb{R}^{d}\right)$ for $0<p \delta<\eta(p)$. Clearly, $f \in B_{p}^{\delta}\left(\mathbb{R}^{d}, e_{i}\right)$ for all $i \in \mathcal{D}$. Let $p \beta_{1}<\eta(p, e)$. Since $f \in B_{p}^{\beta_{1}}\left(\mathbb{R}^{d}, e\right)$, then Definition 2 and the partial ordering property (15) yield the third point. 
2.2. Connection Between the Directional Scaling Function and Anisotropic Scaling Functions

Space $B_{p}^{\left(s_{1}, \cdots, s_{d}\right)}\left(\mathbb{R}^{d}\right)$ is related to the anisotropic Besov space $B_{p, \infty}^{\hat{s},\left(\alpha_{1}, \ldots, \alpha_{d}\right)}\left(\mathbb{R}^{d}\right)$ introduced in $[60,61]$ using an anisotropic Littlewood Paley analysis. We will drop $\infty$. Let $\alpha=\left(\alpha_{1}, \ldots, \alpha_{d}\right) \in \mathbb{R}^{d}$ be an anisotropy, i.e.,

$$
\alpha_{1}>0, \cdots, \alpha_{d}>0 \text { and } \sum_{i \in \mathcal{D}} \alpha_{i}=d
$$

For $r>0$ and $x=\left(x_{1}, \ldots, x_{d}\right) \in \mathbb{R}^{d}$, we define the anisotropic map:

$$
r^{\alpha} x=\left(r^{\alpha_{1}} x_{1}, \ldots, r^{\alpha_{d}} x_{d}\right) .
$$

Define the mean smoothness $\hat{s}$ of $\left(s_{1}, \cdots, s_{d}\right)$ and the anisotropic indices by:

$$
\frac{1}{\hat{s}}=\frac{1}{d} \sum_{i \in \mathcal{D}} \frac{1}{s_{i}} \quad \text { and } \quad \alpha_{i}=\frac{\hat{s}}{s_{i}} .
$$

Then,

$$
B_{p}^{\left(s_{1}, \cdots, s_{d}\right)}\left(\mathbb{R}^{d}\right)=B_{p}^{\hat{s}, \alpha}\left(\mathbb{R}^{d}\right) .
$$

If $s_{1}, \cdots, s_{d}=s$ then $\hat{s}=s$ and $\alpha$ is the isotropy $(1, \ldots, 1)$. Thus,

$$
B_{p}^{(s, \cdots, s)}\left(\mathbb{R}^{d}\right)=B_{p}^{s}\left(\mathbb{R}^{d}\right)=B_{p}^{s,(1, \ldots, 1)}\left(\mathbb{R}^{d}\right) .
$$

For a fixed anisotropy $\alpha$, define the anisotropic scaling function by:

$$
\eta_{\alpha}(p)=\sup \left\{\tau p: f \in B_{p}^{\tau, \alpha}\left(\mathbb{R}^{d}\right)\right\} .
$$

Clearly, using relation (21), the substitute $\breve{\eta}(p, e)$ for $\eta(p, e)$ given in (16) satisfies the following result.

Theorem 1. Let $\mathcal{B}$ denotes any orthonormal basis of $\mathbb{R}^{d}$ starting with e. Let $\Omega$ be the set of all anisotropies $\alpha$ satisfying (18) and $\alpha_{2}=\cdots=\alpha_{d}$. Then:

$$
\breve{\eta}(p, e)=\sup _{\alpha \in \Omega}\left(\frac{\eta_{\alpha}(p)}{\alpha_{1}}\right) .
$$

If $\eta(p)>0$ then:

$$
\eta(p, e)=\sup _{\alpha \in \Omega}\left(\frac{\eta_{\alpha}(p)}{\alpha_{1}}\right)
$$

\section{Criteria of Directional Scaling Function}

\subsection{Criterion of Directional Scaling Function in Triebel Wavelet Bases}

We will use Theorem 1 to characterize the directional scaling function $\eta(p, e)$ in Triebel anisotropic wavelet bases $[69,70]$.

Triebel anisotropic wavelets characterize anisotropic Besov spaces; if $\psi_{-1}$ and $\psi_{1}$ are the Lemarié-Meyer [88,89] (respective to Daubechies [90]) father and mother wavelets in the Schwartz class (respective to compactly supported and finitely smooth with a large enough smoothness), such that all moments (respective to a large enough finite number of moments) of $\psi_{1}$ vanish, $\int_{\mathbb{R}} \psi_{-1}(x) d x=1$ and the collection $\left(\psi_{-1}(x-k)\right)_{k \in \mathbb{Z}}$ and $\left(2^{j / 2} \psi_{1}\left(2^{j} x-k\right)\right)_{j \in \mathbb{N}_{0}, k \in \mathbb{Z}}$ is an orthonormal basis of $L^{2}(\mathbb{R})$. Let $\alpha$ 
an anisotropy as in (18). For $j \in \mathbb{N}_{0}$, let $I_{j, \alpha}$ be the set of pairs $(G, l)$ where $G=\left(G_{1}, \ldots, G_{d}\right) \in\{-1,1\}^{d}$ such that at least one component $G_{i}$ is -1 and $\boldsymbol{l}=\left(l_{1}, \ldots, l_{d}\right) \in \mathbb{N}_{0}^{d}$ where:

$$
\begin{gathered}
l_{i}=\left[j \alpha_{i}\right] \text { if } G_{i}=-1, \\
{\left[j \alpha_{i}\right] \leq l_{i}<\left[(j+1) \alpha_{i}\right] \text { if } G_{i}=1 \text { and }\left[(j+1) \alpha_{i}\right]>\left[j \alpha_{i}\right],}
\end{gathered}
$$

and

$$
l_{i}=\left[j \alpha_{i}\right] \text { if } G_{i}=1 \text { and }\left[(j+1) \alpha_{i}\right]=\left[j \alpha_{i}\right] .
$$

(Clearly, in the isotropic setting $\alpha=(1, \ldots, 1)$ and $l=(j, \ldots, j)$ ).

The cardinality of $I_{j, \alpha}$ is bounded independently of $j$, more precisely:

$$
1 \leq \sharp I_{j, \alpha} \leq\left(2^{d}-1\right) \prod_{i \in \mathcal{D}}\left(2+\alpha_{i}\right) .
$$

The following proposition is given in $[69,70]$.

Proposition 2. For all $x=\left(x_{1}, \ldots, x_{d}\right) \in \mathbb{R}^{d}$ and all $k=\left(k_{1}, \ldots, k_{d}\right) \in \mathbb{Z}^{d}$, we put:

$$
\Psi_{-1, \cdots,-1, k}(x):=\prod_{i \in \mathcal{D}} \psi_{-1}\left(x_{i}-k_{i}\right),
$$

and:

$$
\Psi_{j, k, \alpha}^{(G, l)}(x)=\prod_{i \in \mathcal{D}} \psi_{G_{i}}\left(2^{l_{i}} x_{i}-k_{i}\right)
$$

Set $|\boldsymbol{l}|:=\sum_{i \in \mathcal{D}} l_{i}$. The collection of the union of $\left(\Psi_{-1, \cdots,-1, k}\right)$ for $k \in \mathbb{Z}^{d}$ and $\left(2^{|l| / 2} \Psi_{j, k, \alpha}^{(G, l)}\right)$ for $j \in \mathbb{N}_{0}$, $(G, l) \in I_{j, \alpha}$ and $k \in \mathbb{Z}^{d}$, is then an orthonormal basis of $L^{2}\left(\mathbb{R}^{d}\right)$. Thus any function $f \in L^{2}\left(\mathbb{R}^{d}\right)$ can be written as:

$$
\begin{aligned}
f(x) & =\sum_{k \in \mathbb{Z}^{d}} c_{-1, \cdots,-1, k} \Psi_{-1, \cdots,-1, k}(x), \\
& +\sum_{j \in \mathbb{N}_{0}} \sum_{k \in \mathbb{Z}^{d}} \sum_{(G, l) \in I_{j, \alpha}} c_{j, k, \alpha}^{(G, l)} \Psi_{j, k, \alpha}^{(G, l)}(x),
\end{aligned}
$$

with:

$$
c_{-1, \cdots,-1, k}=\int_{\mathbb{R}^{d}} f(x) \Psi_{-1, \cdots,-1, k}(x) d x,
$$

and:

$$
c_{j, k, \alpha}^{(G, l)}=2^{|l|} \int_{\mathbb{R}^{d}} f(x) \Psi_{j, k, \alpha}^{(G, l)}(x) d x .
$$

The following result was obtained in $[69,70]$.

Proposition 3. Let $p>0$ and $s \in \mathbb{R}$. Then, $f \in B_{p}^{s, \alpha}\left(\mathbb{R}^{d}\right)$ if and only if:

$$
\sup _{j \in \mathbb{N}_{0}} 2^{(s p-d) j} \sum_{k \in \mathbb{Z}^{d}} \sum_{(G, l) \in I_{j, \alpha}}\left|c_{j, k, \alpha}^{(G, l)}\right|^{p}<\infty
$$

Using (25), we deduce the following theorem. 
Theorem 2. The anisotropic Besov exponent is given by:

$$
\eta_{\alpha}(p)=\liminf _{j \rightarrow \infty} \frac{\log \left(2^{-d j} \sum_{k \in \mathbb{Z}^{d}} \sum_{(G, l) \in I_{j, \alpha}}\left|c_{j, k, \alpha}^{(G, l)}\right|^{p}\right)}{\log \left(2^{-j}\right)} .
$$

Let $\mathcal{B}$ and $\Omega$ be as in Theorem 1. If $\eta(p)>0$ then:

$$
\eta(p, e)=\sup _{\alpha \in \Omega}\left(\liminf _{j \rightarrow \infty} \frac{\log \left(2^{-d j} \sum_{k \in \mathbb{Z}^{d}(G, l) \in I_{j, \alpha}}\left|c_{j, k, \alpha}^{(G, l)}\right|^{p}\right)}{\log \left(2^{-j \alpha_{1}}\right)}\right) .
$$

\subsection{Criterion of Directional Scaling Function in Hyperbolic Wavelet Bases}

We will use Theorem 1 to characterize the directional scaling function $\eta(p, e)$ in hyperbolic wavelet bases [68,73-76]. As in [39], without any loss of generality take $d=2$. For $x=\left(x_{1}, x_{2}\right) \in \mathbb{R}^{2}$ and $k=\left(k_{1}, k_{2}\right) \in \mathbb{Z}^{2}$, we put $\Psi_{-1,-1, k}(x)$ as in (30) for $d=2$,

$$
\begin{aligned}
& \Psi_{j_{1}, j_{2}, k}(x)=\psi_{1}\left(2^{j_{1}} x_{1}-k_{1}\right) \psi_{1}\left(2^{j_{2}} x_{2}-k_{2}\right), \\
& \Psi_{j_{1},-1, k}(x)=\psi_{1}\left(2^{j_{1}} x_{1}-k_{1}\right) \psi_{-1}\left(x_{2}-k_{2}\right), \\
& \Psi_{-1, j_{2}, k}(x)=\psi_{-1}\left(x_{1}-k_{1}\right) \psi_{1}\left(2^{j_{2}} x_{2}-k_{2}\right) .
\end{aligned}
$$

The collection of the union of $\left\{\Psi_{-1,-1, k}: k \in \mathbb{Z}^{2}\right\},\left\{2^{\left(j_{1}+j_{2}\right) / 2} \Psi_{j_{1}, j_{2}, k}:\left(j_{1}, j_{2}\right) \in \mathbb{N}_{0}^{2}, k \in \mathbb{Z}^{2}\right\}$, $\left\{2^{j_{1} / 2} \Psi_{j_{1},-1, k}: j_{1} \in \mathbb{N}_{0}, k \in \mathbb{Z}^{2}\right\}$, and $\left\{2^{j_{2} / 2} \Psi_{-1, j_{2}, k}: j_{2} \in \mathbb{N}_{0}, k \in \mathbb{Z}^{2}\right\}$, is then an orthonormal basis of $L^{2}\left(\mathbb{R}^{2}\right)$. Thus any function $f \in L^{2}\left(\mathbb{R}^{2}\right)$ can be written as:

$$
\begin{aligned}
f(x) & =\sum_{k \in \mathbb{Z}^{2}} c_{-1,-1, k} \Psi_{-1,-1, k}(x)+\sum_{\left(j_{1}, j_{2}\right) \in \mathbb{N}_{0}^{2}} \sum_{k \in \mathbb{Z}^{2}} c_{j_{1}, j_{2}, k} \Psi_{j_{1}, j_{2}, k}(x), \\
& +\sum_{j_{1} \in \mathbb{N}_{0}} \sum_{k \in \mathbb{Z}^{2}} c_{j_{1},-1, k} \Psi_{j_{1},-1, k}(x)+\sum_{j_{2} \in \mathbb{N}_{0}} \sum_{k \in \mathbb{Z}^{2}} c_{-1, j_{2}, k} \Psi_{-1, j_{2}, k}(x),
\end{aligned}
$$

with $c_{-1,-1, k}$ as in (34) for $d=2$,

$$
\begin{gathered}
c_{j_{1}, j_{2}, k}=2^{\left(j_{1}+j_{2}\right)} \int_{\mathbb{R}^{2}} f(x) \Psi_{j_{1}, j_{2}, k}(x) d x, \\
c_{j_{1},-1, k}=2^{j_{1}} \int_{\mathbb{R}^{2}} f(x) \Psi_{j_{1},-1, k}(x) d x,
\end{gathered}
$$

and:

$$
c_{-1, j_{2}, k}=2^{j_{2}} \int_{\mathbb{R}^{2}} f(x) \Psi_{-1, j_{2}, k}(x) d x .
$$

Let $\left(\alpha_{1}, \alpha_{2}\right)$ an anisotropy as in (18) (with $d=2$ ). For $j \in \mathbb{N}_{0}$, set:

$$
\Gamma_{j}\left(\alpha_{1}, \alpha_{2}\right)=\Gamma_{j}^{h, l}\left(\alpha_{1}, \alpha_{2}\right) \cup \Gamma_{j}^{l, h}\left(\alpha_{1}, \alpha_{2}\right) \cup \Gamma_{j}^{h, h}\left(\alpha_{1}, \alpha_{2}\right),
$$

with

$$
\begin{gathered}
\Gamma_{j}^{h, h}\left(\alpha_{1}, \alpha_{2}\right)=\prod_{i=1}^{2}\left\{\left[(j-1) \alpha_{i}\right]-1, \cdots,\left[j \alpha_{i}\right]+1\right\}, \\
\Gamma_{j}^{h, l}\left(\alpha_{1}, \alpha_{2}\right)=\left\{\left[(j-1) \alpha_{1}\right]-1, \cdots,\left[j \alpha_{1}\right]+1\right\} \times\left\{0, \cdots,\left[(j-1) \alpha_{2}\right]-1\right\},
\end{gathered}
$$


and

$$
\Gamma_{j}^{l, h}\left(\alpha_{1}, \alpha_{2}\right)=\left\{0, \cdots,\left[(j-1) \alpha_{1}\right]-1\right\} \times\left\{\left[(j-1) \alpha_{2}\right]-1, \cdots,\left[j \alpha_{2}\right]+1\right\} .
$$

The following result was obtained in [39].

Proposition 4. Let $p>0$ and $s \in \mathbb{R}$. Then, $f \in B_{p}^{s,\left(\alpha_{1}, \alpha_{2}\right)}\left(\mathbb{R}^{2}\right)$ if and only if:

$$
\sup _{j \in \mathbb{N}_{0}} \sup _{\left(j_{1}, j_{2}\right) \in \Gamma_{j}\left(\alpha_{1}, \alpha_{2}\right)} 2^{s p j-\left(j_{1}+j_{2}\right)} \sum_{k \in \mathbb{Z}^{2}}\left|c_{j_{1}, j_{2}, k}\right|^{p}<\infty
$$

Using (25), we deduce the following theorem.

Theorem 3. The anisotropic Besov exponent is given by:

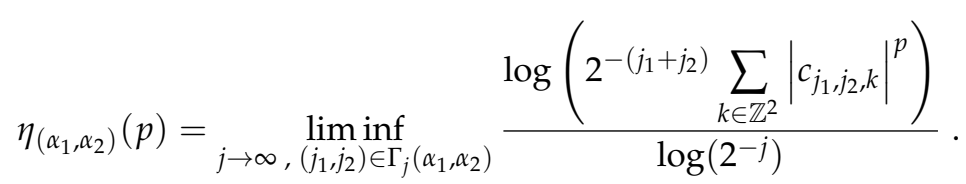

Let $\mathcal{B}$ and $\Omega$ be as in Theorem 1. If $\eta(p)>0$ then:

$$
\eta(p, e)=\sup _{\left(\alpha_{1}, \alpha_{2}\right) \in \Omega}\left(\liminf _{j \rightarrow \infty,\left(j_{1}, j_{2}\right) \in \Gamma_{j}\left(\alpha_{1}, \alpha_{2}\right)} \frac{\log \left(2^{-\left(j_{1}+j_{2}\right)} \sum_{k \in \mathbb{Z}^{2}}\left|c_{j_{1}, j_{2}, k}\right|^{p}\right)}{\log \left(2^{-\alpha_{1} j}\right)}\right) .
$$

\subsection{Criterion of Directional Lipschitz Scaling Function of $f$ on Hyperbolic Schauder Bases}

We will characterize the directional Lipschitz scaling function restricted to a bounded domain on hyperbolic Schauder functions without passing through anisotropies as done previously. Without any loss of generality, we will work on the unit cube $I^{d}$. For this purpose we have to adapt the difference $\Delta_{t, e} f$ in direction $e$ by the standard formula

$$
\Delta_{t, e} f(y)= \begin{cases}f(y+t e)-f(y) & \text { if } y+t e \in I^{d} \\ 0 & \text { if } y+t e \notin I^{d}\end{cases}
$$

Definition 3. Let $0<s<1,1 \leq p<\infty$ and $f \in L^{p}\left(I^{d}\right)$. We say that $f \in \operatorname{Lip}_{p}^{s}\left(I^{d}, e\right)$ if there exists $C>0$ such that:

$$
\forall 0<t \leq 1 \quad \int_{I^{d}}\left|\Delta_{t, e} f(y)\right|^{p} d y \leq C|t|^{s p} .
$$

Define the directional Lipschitz scaling function of $f$ in direction e by:

$$
\eta_{L}(p, e)=p \sup \left\{0<s<1: f \in \operatorname{Lip} p_{p}^{s}\left(I^{d}, e\right)\right\} .
$$

We say that $f \in \operatorname{Lip}_{p}^{s}\left(I^{d}\right)$ if there exists $C>0$ such that:

$$
\forall e \in S^{d-1} \forall 0<t \leq 1 \quad \int_{I^{d}}\left|\Delta_{t, e} f(y)\right|^{p} d y \leq C|t|^{s p} .
$$

Define the Lipschitz scaling function of $f$ by:

$$
\eta_{L}(p)=p \sup \left\{0<s<1: f \in \operatorname{Lip}_{p}^{s}\left(I^{d}\right)\right\} .
$$


Remark 2. Fix $1 \leq p<\infty$. As in Bonami and Estrade [55], using triangular inequality, we can prove that if there exists $e_{0}$ such that $0<\eta_{L}\left(p, e_{0}\right)<p$ then the map $e \mapsto \eta_{L}(p, e)$ takes at most $d$ different values. Moreover, it is constant except, perhaps, on the intersection of unit sphere $S^{d-1}$ with a subspace of dimension at most $d-1$ where it may take larger values. Therefore,

$$
\eta_{L}(p)=\min _{e \in S^{d-1}} \eta_{L}(p, e)=\min _{i \in \mathcal{D}} \eta_{L}\left(p, e_{i}\right) .
$$

Since we will use a Kamont result [67], we follow the same notations. Let $e_{i}$ and $\mathcal{D}$ be as in Definition 2. Write $\Delta_{t, i} f$ instead of $\Delta_{t, e_{i}} f$. Denote by $\mathbf{0}$ and $\mathbf{1}$, respectively, the vectors $(0, \cdots, 0)$ and $(1, \cdots, 1)$ in $\mathbb{R}^{d}$. Let $\mathbf{a}=\left(a_{1}, \cdots, a_{d}\right)$ and $\mathbf{b}=\left(b_{1}, \cdots, b_{d}\right)$ be two vectors of $\mathbb{R}^{d}$. Put $|\mathbf{a}|=\left|a_{1}\right|+\cdots+\left|a_{d}\right|$. If $A \subset \mathcal{D}$, put $\mathbf{a}(A)=\left(\tilde{a}_{1}, \cdots, \tilde{a}_{d}\right)$ where $\tilde{a}_{i}=a_{i}$ if $i \in A$, and $\tilde{a}_{i}=0$ if $i \notin A$. Write $\mathbf{a} \leq \mathbf{b}$ if $a_{i} \leq b_{i}$ for all $i \in \mathcal{D}$, and $\mathbf{a}<\mathbf{b}$ if $a_{i}<b_{i}$ for all $i \in \mathcal{D}$. Finally, write $\mathbf{a}^{\mathbf{b}}=\prod_{i \in \mathcal{D}} a_{i}^{b_{i}}$. For $\mathbf{h}=\left(h_{1}, \cdots, h_{d}\right) \in \mathbb{R}^{d}$ and $A=\left\{i_{1}, \cdots, i_{k}\right\} \subset \mathcal{D}$, set:

$$
\Delta_{\mathbf{h}, A} f=\Delta_{h_{i_{1}, i_{1}} \circ \cdots \circ \Delta_{h_{i_{k}}, i_{k}} f} .
$$

Clearly,

$$
\Delta_{h_{i}, i} \circ \Delta_{h_{j}, j} f=\Delta_{h_{j}, j} \circ \Delta_{h_{i}, i} f .
$$

For $f \in L^{p}\left(I^{d}\right)$, define:

$$
\omega_{p, A}(f, \mathbf{t})=\sup _{\mathbf{0}<\mathbf{h} \leq \mathbf{t}}\left\|\Delta_{\mathbf{h}, A} f\right\|_{p} \quad \text { for } \quad \mathbf{t} \in \mathbb{R}^{d}, \quad \mathbf{0}<\mathbf{t} \leq \mathbf{1}
$$

Remark 3. Clearly, $f \in \operatorname{Lip}_{p}^{s_{i}}\left(I^{d}, e_{i}\right)$ is equivalent to $\omega_{p,\{i\}}(f, \mathbf{t})=O\left(t_{i}^{s_{i}}\right)$.

Let $\mathbf{0}<\left(s_{1}, \cdots, s_{d}\right)<\mathbf{1}$. For $\mathbf{t}=\left(t_{1}, \cdots, t_{d}\right)$, define:

$$
\omega^{\left(s_{1}, \cdots, s_{d}\right)}(\mathbf{t})=\prod_{i \in \mathcal{D}} t_{i}^{s_{i}}
$$

and

$$
\omega^{\left(s_{1}, \cdots, s_{d}\right), \frac{1}{2}}(\mathbf{t})=\left(\prod_{i \in \mathcal{D}} t_{i}^{s_{i}}\right)\left(1-\sum_{i \in \mathcal{D}} \log \left(t_{i}\right)\right)^{1 / 2} .
$$

For a function $g$ given on $I^{d}, A \subset \mathcal{D}$, and $\mathbf{t} \in I^{d}$, put:

$$
g(\mathbf{t} ; A)=g(\mathbf{t}(A)+\mathbf{1}(\mathcal{D} \backslash A)) .
$$

Set:

$$
\mathcal{D}^{*}=\{A \subset \mathcal{D}: A \neq \varnothing\} .
$$

In [67], Kamont considered the following spaces described in terms of moduli of smoothness in the $L^{p}$-norm:

$$
\begin{gathered}
\operatorname{Lip}_{p}^{\left(s_{1}, \cdots, s_{d}\right)}\left(I^{d}\right)=\left\{f \in L^{p}\left(I^{d}\right): \forall A \in \mathcal{D}^{*} \omega_{p, A}(f, \mathbf{t})=O\left(\omega^{\left(s_{1}, \cdots, s_{d}\right)}(\mathbf{t} ; A)\right)\right\}, \\
\operatorname{Lip}_{p}^{\left(s_{1}, \cdots, s_{d}\right), \frac{1}{2}}\left(I^{d}\right)=\left\{f \in L^{p}\left(I^{d}\right): \forall A \in \mathcal{D}^{*} \omega_{p, A}(f, \mathbf{t})=O\left(\omega^{\left(s_{1}, \cdots, s_{d}\right), \frac{1}{2}}(\mathbf{t} ; A)\right)\right\},
\end{gathered}
$$

and

$$
\operatorname{lip}_{p}^{\left(s_{1}, \cdots, s_{d}\right), \frac{1}{2}}\left(I^{d}\right)=\left\{f \in \operatorname{Lip}_{p}^{\left(s_{1}, \cdots, s_{d}\right), \frac{1}{2}}\left(I^{d}\right): \forall A \in \mathcal{D}^{*} \omega_{p, A}(f, \mathbf{t})=o\left(\omega^{\left(s_{1}, \cdots, s_{d}\right), \frac{1}{2}}(\mathbf{t} ; A)\right)\right\},
$$

where $O(\mathbf{t}(A))$ and $o(\mathbf{t}(A))$ refer to $\min \left(t_{i}: i \in A\right) \rightarrow 0$. 
The following embeddings hold.

Proposition $5 . \quad 1$.

$$
\operatorname{Lip}_{p}^{\left(s_{1}, \cdots, s_{d}\right)}\left(I^{d}\right) \subset \bigcap_{i \in \mathcal{D}} \operatorname{Lip}_{p}^{s_{i}}\left(I^{d}, e_{i}\right)
$$

2.

$$
\bigcap_{i \in \mathcal{D}} \operatorname{Lip}_{p}^{s_{i}}\left(I^{d}, e_{i}\right) \subset \operatorname{Lip}_{p}^{\left(\theta_{1} s_{1}, \cdots, \theta_{d} s_{d}\right)}\left(I^{d}\right) \quad \forall \mathbf{0}<\theta=\left(\theta_{1}, \cdots, \theta_{d}\right) \text { with }|\theta| \leq 1
$$

\section{Proof of Proposition 5:}

1. If $f \in \operatorname{Lip} p_{p}^{\left(s_{1}, \cdots, s_{d}\right)}\left(I^{d}\right)$, then $\omega_{p,\{i\}}(f, \mathbf{t})=O\left(t_{i}^{s_{i}}\right)$ for all $i \in \mathcal{D}$. The result follows from Remark 3 .

2. Conversely, assume that $f \in \operatorname{Lip}_{p}^{s_{i}}\left(I^{d}, e_{i}\right)$ for all $i \in \mathcal{D}$. Let $A \subset \mathcal{D}$ be non-empty. Write $A=\left\{i_{1}, \cdots, i_{k}\right\}$, we have $\Delta_{h, A} f=\Delta_{h_{i_{1}}, i_{1}} g$ where $g=\Delta_{h_{i_{2}}, i_{2}} \circ \cdots \circ \Delta_{h_{i_{k}}, i_{k}} f$. Since $f \in \operatorname{Lip}_{p}^{s_{i}}\left(I^{d}, e_{i}\right)$ and $g$ is a linear combination of translated copies of $f$, then $\omega_{p, A}(f, \mathbf{t})=O\left(t_{i_{1}}^{s_{i_{1}}}\right)$. Similarly, using property (58), we have $\omega_{p, A}(f, \mathbf{t})=O\left(t_{i_{l}}^{s_{i}}\right)$ for all $2 \leq l \leq k$. On the other hand, since $f \in L^{p}\left(I^{d}\right)$ then $\omega_{A}(f, \mathbf{t})=O(1)$ for all $k+1 \leq l \leq d$. Therefore, (68) holds.

The following embeddings hold too.

Proposition 6. 1. We have $\operatorname{Lip}_{p}^{\left(s_{1}, \cdots, s_{d}\right)}\left(I^{d}\right) \subset \operatorname{Lip}_{p}^{\left(s_{1}, \cdots, s_{d}\right), \frac{1}{2}}\left(I^{d}\right)$ and $\operatorname{lip}_{p}^{\left(s_{1}, \cdots, s_{d}\right)}\left(I^{d}\right) \subset \operatorname{lip}_{p}^{\left(s_{1}, \cdots, s_{d}\right), \frac{1}{2}}\left(I^{d}\right)$.

2. If $\left(s_{1}^{\prime}, \cdots, s_{d}^{\prime}\right)<\left(s_{1}, \cdots, s_{d}\right)$ then $\operatorname{Lip}_{p}^{\left(s_{1}, \cdots, s_{d}\right)}\left(I^{d}\right) \subset \operatorname{lip}_{p}^{\left(s_{1}^{\prime}, \cdots, s_{d}^{\prime}\right)}\left(I^{d}\right)$.

3. If $\left(s_{1}^{\prime}, \cdots, s_{d}^{\prime}\right)<\left(s_{1}, \cdots, s_{d}\right)$ then $\operatorname{Lip}_{p}^{\left(s_{1}, \cdots, s_{d}\right), \frac{1}{2}}\left(I^{d}\right) \subset \operatorname{Lip}_{p}^{\left(s_{1}^{\prime}, \cdots, s_{d}^{\prime}\right)}\left(I^{d}\right)$.

\section{Proof of Proposition 6:}

1. The first point is a consequence of $\omega^{\left(s_{1}, \cdots, s_{d}\right)}(\mathbf{t}) \leq \omega^{\left(s_{1}, \cdots, s_{d}\right), \frac{1}{2}}(\mathbf{t})$.

2. Let $f \in \operatorname{Lip}_{p}^{\left(s_{1}, \cdots, s_{d}\right)}\left(I^{d}\right)$ and $\left(s_{1}^{\prime}, \cdots, s_{d}^{\prime}\right)<\left(s_{1}, \cdots, s_{d}\right)$. We know from (69) that $f \in \operatorname{Lip}_{p}^{\left(s_{1}^{\prime}, \cdots, s_{d}^{\prime}\right)}\left(I^{d}\right)$. Let $A \in \mathcal{D}^{*}$. Since $\left(s_{1}^{\prime}, \cdots, s_{d}^{\prime}\right)<\left(s_{1}, \cdots, s_{d}\right)$ then

$$
\frac{\omega_{A}(f, \mathbf{t})}{\omega^{\left(s_{1}^{\prime}, \cdots, s_{d}^{\prime}\right)}(\mathbf{t} ; A)} \leq C \omega^{\left(s_{1}, \cdots, s_{d}\right)-\left(s_{1}^{\prime}, \cdots, s_{d}^{\prime}\right)}(\mathbf{t} ; A)=o(\mathbf{t}(A)) .
$$

Hence $f \in \operatorname{lip}_{p}^{\left(s_{1}^{\prime}, \cdots, s_{d}^{\prime}\right)}\left(I^{d}\right)$.

3. Let $f \in \operatorname{Lip}_{p}^{\left(s_{1}, \cdots, s_{d}\right), \frac{1}{2}}\left(I^{d}\right)$ and $\left(s_{1}^{\prime}, \cdots, s_{d}^{\prime}\right)<\left(s_{1}, \cdots, s_{d}\right)$. Let $A \in \mathcal{D}^{*}$. Since $\left(s_{1}^{\prime}, \cdots, s_{d}^{\prime}\right)<$ $\left(s_{1}, \cdots, s_{d}\right)$ and $t \log t=o(1)$ when $t$ goes to 0 then

$$
\frac{\omega_{A}(f, \mathbf{t})}{\omega^{\left(s_{1}^{\prime}, \cdots, s_{d}^{\prime}\right)}(\mathbf{t} ; A)} \leq C \omega^{\left(s_{1}, \cdots, s_{d}\right)-\left(s_{1}^{\prime}, \cdots, s_{d}^{\prime}\right)}(\mathbf{t} ; A)\left(1-\sum_{i \in A} \log \left(t_{i}\right)\right)^{1 / 2}=o(\mathbf{t}(A)) .
$$

It follows that $f \in \operatorname{Lip} p^{\left(s_{1}^{\prime}, \cdots, s_{d}^{\prime}\right)}\left(I^{d}\right)$.

We will characterize $\eta_{L}(p, e)$ in terms of decay conditions for the coefficients of the expansion of $f$ in the basis of tensor products of Schauder functions. Without any loss of generality, the orthonormal basis $\mathcal{B}$ (on which coordinates are considered) can start with $e$.

Using the partial ordering property

$$
\operatorname{Lip}_{p}^{\left(s_{1}, \cdots, s_{d}\right)}\left(I^{d}\right) \subset \operatorname{Lip}_{p}^{\left(s_{1}^{\prime}, \cdots, s_{d}^{\prime}\right)}\left(I^{d}\right) \quad \forall\left(s_{1}^{\prime}, \cdots, s_{d}^{\prime}\right) \leq\left(s_{1}, \cdots, s_{d}\right),
$$

we introduce the following definition as a substitute for $\eta_{L}(p, e)$

$$
\widetilde{\eta_{L}}(p, e)=p \quad \sup \left\{s_{1} \in(0,1): \exists 0<\varepsilon<1 \quad f \in \operatorname{Lip}_{p}^{\left(s_{1}, \varepsilon, \cdots, \varepsilon\right)}\left(I^{d}\right)\right\} .
$$


We will show the following proposition.

Proposition 7. 1. If $\eta_{L}(p, e)=0$ then $\widetilde{\eta_{L}}(p, e)=0$.

2. We have always

$$
\widetilde{\eta_{L}}(p, e) \leq \eta_{L}(p, e)
$$

3. If $\eta_{L}(p)>0$ then $\widetilde{\eta_{L}}(p, e)=\eta_{L}(p, e)$.

\section{Proof of Proposition 7:}

Both first and second results are consequences of the first part of Proposition 5.

Assume that $\eta_{L}(p)>0$, then $f \in \operatorname{Lip}_{p}^{\delta}\left(I^{d}\right)$ for $0<p \delta<\eta_{L}(p)$. Clearly, $f \in \operatorname{Lip}_{p}^{\delta}\left(I^{d}, e_{i}\right)$ for all $i \in \mathcal{D}$ and $\eta_{L}(p, e) \geq p \delta$. Let $p s_{1}<\eta_{L}(p, e)$. Since $f \in L i p_{p}^{s_{1}}\left(I^{d}, e\right)$, then the second result in Proposition 5 implies that $f \in \operatorname{Lip}_{p}^{\left((1-(d-1) \theta) s_{1}, \theta \delta, \cdots, \theta \delta\right)}\left(I^{d}\right)$ for all $0<\theta \leq \frac{1}{d-1}$. Letting $\theta$ tends to 0 , we obtain $\widetilde{\eta_{L}}(p, e) \geq \eta_{L}(p, e)$.

In $[67,87]$, Kamont characterized the space $\operatorname{Lip}_{p}^{\left(s_{1}, \cdots, s_{d}\right)}\left(I^{d}\right)$ in terms of decay conditions for the coefficients of the expansion of $f$ in the basis of tensor products of Schauder functions.

Let $\left\{\phi_{k}, k \geq 0\right\}$ be the family of Schauder functions on $I$, normed in $L^{\infty}$, i.e., $\phi_{0}=1, \phi_{1}(t)=t$, and for $k \geq 2, k=2^{j}+n$ with $j \geq 0$ and $1 \leq n \leq 2^{j}, \phi_{k}(t)=\phi\left(2^{j+1} t-2 n+1\right)$ (with support $\left.\left[(n-1) 2^{-j}, n 2^{-j}\right]\right)$, where $\phi(t)=\max (0,1-|t|)$ (the so-called Schauder function).

In several dimensions, we consider the family $\left\{\Phi_{\mathbf{k}}, \mathbf{k} \geq 0\right\}$ of tensor products of Schauder functions, i.e., $\Phi_{\mathbf{k}}(x)=\phi_{k_{1}}\left(x_{1}\right) \cdots \phi_{k_{d}}\left(x_{d}\right)$ for $\mathbf{k}=\left(k_{1}, \cdots, k_{d}\right)$.

For $j \in M=\{-2,-1,0,1,2, \cdots\}$, let

$$
\tilde{N}_{-2}=\{0\}, \tilde{N}_{-1}=\{1\} \text {, and } \tilde{N}_{j}=\left\{2^{j}+n: n=1, \cdots, 2^{j}\right\} \text { for } j \geq 0,
$$

and for a vector $\mathbf{j}=\left(j_{1}, \cdots, j_{d}\right)$ we put

$$
\tilde{N}_{\mathbf{j}}=\tilde{N}_{j_{1}} \times \cdots \tilde{N}_{j_{d}}
$$

Let for $f \in C\left(I^{d}\right), i \in \mathcal{D}, x \in I^{d}$ and $k \geq 0$

$$
c_{i, 0}(f)(x)=f\left(x-x_{i} e_{i}\right), c_{i, 1}(f)(x)=f\left(x+\left(1-x_{i}\right) e_{i}\right)-f\left(x-x_{i} e_{i}\right),
$$

and for $k=2^{j}+n \in \tilde{N}_{j}$ with $j \geq 0$

$$
c_{i, k}(f)(x)=f\left(x+\left(\frac{2 n-1}{2^{j+1}}-x_{i}\right) e_{i}\right)-\frac{1}{2}\left(f\left(x+\left(\frac{n-1}{2^{j}}-x_{i}\right) e_{i}\right)+f\left(x+\left(\frac{n}{2^{j}}-x_{i}\right) e_{i}\right)\right) .
$$

For $\mathbf{k}=\left(k_{1}, \cdots, k_{d}\right)$ we put

$$
C_{\mathbf{k}}(f)=c_{1, k_{1}} \circ \cdots \circ c_{d, k_{d}}(f)
$$

Then for any $f \in C\left(I^{d}\right)$ we have

$$
f=\sum_{\mathbf{j} \in M^{d}} \sum_{\mathbf{k} \in \tilde{N}_{\mathbf{j}}} C_{\mathbf{k}}(f) \Phi_{\mathbf{k}} .
$$

In $\sum_{\mathbf{j} \in M^{d}}$ we assume the following order: for $\mathbf{j}=\left(j_{l}, \ldots, j_{d}\right)$ and $\mathbf{j}^{\prime}=\left(j_{l}^{\prime}, \ldots, j_{d}^{\prime}\right)$, if $\max \left(j_{1}, \ldots, j_{d}\right)<$ $\max \left(j_{1}^{\prime}, \ldots, j_{d}^{\prime}\right)$, then $\mathbf{j}$ precedes $\mathbf{j}^{\prime}$.

For $f$ given by (77) we put

$$
\tau_{\mathbf{j}, p}(f)=2^{-|\mathbf{j}| / p}\left(\sum_{\mathbf{k} \in \tilde{N}_{\mathbf{j}}}\left|C_{\mathbf{k}}(f)\right|^{p}\right)^{1 / p} .
$$


The following wavelet characterization of spaces $\operatorname{Lip}_{p}^{\left(s_{1}, \cdots, s_{d}\right)}\left(I^{d}\right)$ is due to Kamont [67].

Proposition 8. Let:

$$
\mathbf{t}_{\mathbf{j}}=\left(2^{-\max \left(j_{1}, 0\right)}, \cdots, 2^{-\max \left(j_{d}, 0\right)}\right) .
$$

Then, for $(1 / p, \cdots, 1 / p)<\left(s_{1}, \cdots, s_{d}\right)<\mathbf{1}$,

$$
f \in \operatorname{Lip}_{p}^{\left(s_{1}, \cdots, s_{d}\right)}\left(I^{d}\right) \Leftrightarrow \tau_{\mathbf{j}, p}(f)=O\left(\omega^{\left(s_{1}, \cdots, s_{d}\right)}\left(\mathbf{t}_{\mathbf{j}}\right)\right) \text { as }|\mathbf{j}| \rightarrow \infty
$$

Thanks to Proposition 7, the last result leads to the following characterization.

Theorem 4. Assume that $\eta_{L}(p)>0$. If:

$$
\forall i \in \mathcal{D} \quad \liminf _{|\mathbf{j}| \rightarrow \infty} \frac{\log \tau_{\mathbf{j}, p}(f)}{\log \left(2^{-\max \left(j_{i}, 0\right)}\right)}>1 / p,
$$

then:

$$
\widetilde{\eta_{L}}\left(p, e_{i}\right)=\eta_{L}\left(p, e_{i}\right)=p \min \left(1, \liminf _{|\mathbf{j}| \rightarrow \infty} \frac{\log \tau_{\mathbf{j}, p}(f)}{\log \left(2^{-\max \left(j_{i}, 0\right)}\right)}\right) .
$$

Remark 4. We will see that assumption (81) yields the appropriate range P given in (9) (thanks to (56)) for the directional thermodynamic formalisms that we will find in Section 7 (respective to Section 8) for fractional Brownian sheets (respective to Sierpinski cascade functions).

\section{General Upper Bound for the Directional Hölder Spectrum}

Let us first recall the notions of Hölder regularity, directional Hölder regularity and anisotropic Hölder regularity.

Definition 4. Let $h>0$ be non integer, $y \in \mathbb{R}^{d}$ and $f: \mathbb{R}^{d} \rightarrow \mathbb{C}$. We say that $f \in C^{h}(y)$ if there exists $C>0$ and a polynomial $P_{y}$ of degree at most the integer part $[h]$ of $h$ such that in a neighborhood of $y$ we have

$$
\left|f(x)-P_{y}(x)\right| \leq C|x-y|^{h} .
$$

The Hölder exponent (or regularity) of $f$ at $y$ is

$$
h(y)=\sup \left\{h: \quad f \in C^{h}(y)\right\} .
$$

We say that $f \in C^{h}\left(\mathbb{R}^{d}\right)$ if $f \in L^{\infty}\left(\mathbb{R}^{d}\right)$ and if (82) holds for any $x$ and $y$ in $\mathbb{R}^{d}$ with uniform constant $C$.

The Hölder (upper-Hölder) spectrum of $f$ is the map which associates to each $H$ the Hausdorff dimension $d(H)$ (respective to $D(H)$ ) of the set of points $y$ where $h(y)=H$ (respective to $h(y) \leq H$ ).

Definition 5. Let $h>0$ be non integer, $y \in \mathbb{R}^{d}$ and $f: \mathbb{R}^{d} \rightarrow \mathbb{C}$. Let $e \in S^{d-1}$. We say that $f \in C^{h}(y, e)$ if there exists $\delta>0, C>0$ and a polynomial $P_{y}$ of degree at most the integer part $[h]$ of $h$ such that for all $t \in(-\delta, \delta)$

$$
\left|f(y+t e)-P_{y}(y+t e)\right| \leq C|t|^{h} .
$$

The directional Hölder exponent (or regularity) of $f$ at $y$ in direction $e$ is

$$
h(y, e)=\sup \left\{h: \quad f \in C^{h}(y, e)\right\} .
$$


We say that $f \in C^{h}\left(\mathbb{R}^{d}, e\right)$ if $f \in L^{\infty}\left(\mathbb{R}^{d}\right)$ and if (83) holds for any $y \in \mathbb{R}^{d}$ and $t \in \mathbb{R}$ with uniform constant $C$.

The directional Hölder (upper-Hölder) spectrum of $f$ in direction $e$ is the map which associates to each $H$ the Hausdorff dimension $d(H, e)$ (respective to $D(H, e)$ ) of the set of points $y$ where $h(y, e)=H$ (respective to. $h(y, e) \leq H$ ).

In [44], we found a connection between both the notion of directional Hölder regularity and the anisotropic version of Definition 4 (see $[29,43])$.

Let $\alpha \in \mathbb{R}^{d}$ be an anisotropy as in (18). For $x=\left(x_{1}, \ldots, x_{d}\right) \in \mathbb{R}^{d}$, we set

$$
|x|_{\alpha}=\max \left(\left|x_{1}\right|^{1 / \alpha_{1}}, \ldots,\left|x_{d}\right|^{1 / \alpha_{d}}\right) .
$$

The corresponding $\alpha$-ball $R_{\alpha}(x, r):=\left\{y \in \mathbb{R}^{d}:|x-y|_{\alpha}<r\right\}$ of $\alpha$-radius $r$ centered on $x$ is a rectangle with sides parallel to the axes of coordinates, centered at $x$ and with side-length $2 r^{\alpha_{i}}$ in the $x_{i}$-direction. If $P=\sum_{\left(i_{1}, \ldots, i_{d}\right) \in \mathbb{N}_{0}^{d}} a_{\left(i_{1}, \ldots, i_{d}\right)} x_{1}^{i_{1}} \cdots x_{d}^{i_{d}}$ is a polynomial, define its $\alpha$-homogeneous degree by

$$
d_{\alpha}^{o} P=\max \left\{\sum_{l \in \mathcal{D}} \alpha_{l} i_{l}: a_{\left(i_{1}, \ldots, i_{d}\right)} \neq 0\right\} .
$$

Definition 6. Let $h>0$ and $y \in \mathbb{R}^{d}$. A function $f: \mathbb{R}^{d} \rightarrow \mathbb{C}$ belongs to $C_{\alpha}^{h}(y)$ if there exist $C>0$ and $a$ polynomial $P$ of $\alpha$-homogeneous degree smaller than $h$ such that in a neighborhood of $y$

$$
\left|f(x)-P_{y}(x)\right| \leq C|x-y|_{\alpha}^{h} .
$$

The $\alpha$-Hölder exponent of $f$ at $y$ is defined by:

$$
h_{\alpha}(y)=\sup \left\{h: \quad f \in C_{\alpha}^{h}(y)\right\} .
$$

In [44], we found a connection between both directional and anisotropic pointwise Hölder exponents of $f$.

Proposition 9. Let $\mathcal{B}$ and $\Omega$ be as in Theorem 1. Then

$$
h(x, e)=\sup _{\alpha \in \Omega}\left(\frac{h_{\alpha}(x)}{\alpha_{1}}\right) .
$$

Proposition 9 yields the following general upper bound for the directional Hölder spectrum.

Theorem 5. Let $\mathcal{B}$ and $\Omega$ be as in Theorem 1. Then

$$
D(H, e) \leq \inf _{\alpha \in \Omega} \operatorname{dim}\left\{x \in \mathbb{R}^{d}: h_{\alpha}(x) \leq \alpha_{1} H\right\} .
$$

Remark 5. In [58], we characterized directional pointwise Lipschitz regularity in terms of decay conditions for the coefficients of the expansion of $f$ in the hyperbolic basis of tensor products of Schauder functions (see Section 8.2). Nevertheless, we do not yet deduce a general upper bound for the directional Lipschitz spectrum.

Let us now show how to use Theorem 5 in order to obtain a general upper bound for the directional spectrum. In $[29,43]$, we adapted the notion of Hausdorff dimension to the anisotropy $\alpha$; if $E \subset \mathbb{R}^{d}$, we define its $\alpha$-diameter to be $|E|_{\alpha}:=\sup _{x, y \in E}|x-y|_{\alpha}$. By replacing in the definition of Hausdorff measure, the usual notion of diameter by the $\alpha$-diameter, we easily check (see [91]) that we get the following notion of anisotropic dimension. 
Definition 7. Let $E \subset \mathbb{R}^{d}, \varepsilon>0$ and $R_{\varepsilon}$ the set of all coverings $R=\left(E_{n}\right)_{n \in \mathbb{N}}$ of $E$ by sets $E_{n}$ of $\alpha$-diameter $\left|E_{n}\right|_{\alpha}$ at most $\varepsilon$. Let

$$
M_{\varepsilon, \alpha}^{\delta}(E)=\inf _{R \in R_{\varepsilon}} \sum_{n \in \mathbb{N}}\left|E_{n}\right|_{\alpha}^{\delta} .
$$

The $\delta$-dimensional $\alpha$-Hausdorff measure of $E$ is

$$
M_{\alpha}^{\delta}(E)=\limsup _{\varepsilon \rightarrow 0} M_{\varepsilon, \alpha}^{\delta}(E)
$$

The $\alpha$-Hausdorff dimension of $E$ is

$$
\operatorname{dim}_{\alpha}(E)=\inf \left\{\delta: M_{\alpha}^{\delta}(E)=0\right\}=\sup \left\{\delta: M_{\alpha}^{\delta}(E)=\infty\right\} .
$$

Note that we get the same value of $\operatorname{dim}_{\alpha}(E)$ if we use coverings $R=\left(E_{n}\right)_{n \in \mathbb{N}}$ of $E$ by rectangles $E_{n}$ with sides parallel to the axes of coordinates and with side-length $2 \varepsilon^{\alpha_{i}}$ in the $x_{i}$-direction.

In the isotropic case, $|\cdot|_{(1, \ldots, 1)}$ is equivalent to the Euclidean norm on $\mathbb{R}^{d}$ and $\operatorname{dim}_{(1, \ldots, 1)}(E)$ coincides with $\operatorname{dim} E$. But if $\alpha \neq(1, \ldots, 1)$, then $\operatorname{dim}_{\alpha}(E)$ doesn't necessarily coincide with $\operatorname{dim} E$. Actually, if

$$
\alpha_{\min }=\min _{i \in \mathcal{D}} \alpha_{i} \text { and } \quad \alpha_{\max }=\max _{i \in \mathcal{D}} \alpha_{i}
$$

then there exists $C \geq 1$ such that

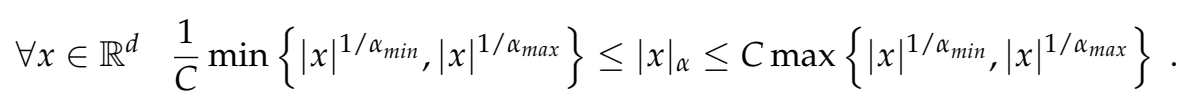

and

$$
\alpha_{\min } \operatorname{dim}(E) \leq \operatorname{dim}_{\alpha}(E) \leq \alpha_{\max } \operatorname{dim}(E)
$$

Definition 8. The $\alpha$-spectrum is:

$$
d_{\alpha}(H)=\operatorname{dim}_{\alpha}\left\{x \in \mathbb{R}^{d}: h_{\alpha}(x)=H\right\} .
$$

The $\alpha$-upper-spectrum is:

$$
D_{\alpha}(H)=\operatorname{dim}_{\alpha}\left\{x \in \mathbb{R}^{d}: h_{\alpha}(x) \leq H\right\} .
$$

The following upper bound was proved in [59].

Proposition 10. If $f$ is uniform Hölder on $\mathbb{R}^{d}$ in the sense that $f \in C^{\varepsilon}\left(\mathbb{R}^{d}\right)$ for $\varepsilon>0$, then:

$$
D_{\alpha}(H) \leq \inf _{p \geq p_{\alpha}}\left(H p-\eta_{\alpha}(p)+d\right),
$$

where $\eta_{\alpha}$ is the anisotropic scaling function of $f$ given in (23) and $p_{\alpha}$ satisfies

$$
\eta_{\alpha}\left(p_{\alpha}\right)=d \text {. }
$$

Consequently, we obtain the following result.

Theorem 6. Let $\mathcal{B}$ and $\Omega$ be as in Theorem 1. If $f$ is uniform Hölder on $\mathbb{R}^{d}$, then

$$
D(H, e) \leq \inf _{\alpha \in \Omega} \frac{1}{\alpha_{\min }} \inf _{p \geq p_{\alpha}}\left(\alpha_{1} H p-\eta_{\alpha}(p)+d\right) .
$$


Proof. By (91)

$$
D(H, e) \leq \inf _{\alpha \in \Omega} \frac{1}{\alpha_{\min }} D_{\alpha}\left(\alpha_{1} H\right)
$$

Thus (94) yields (96).

Remark 6. Let us come back to the optimality of (91). For that, we will consider a general anisotropic Sierpinski carpet; let $s$ and $t$ be two integers with $s \leq t$. We divide the unit square $\mathfrak{R}=[0,1]^{2}$ into a uniform grid of rectangles of height $1 / t$ and width $1 / s$. Choose $A \subset\{0,1, \ldots, s-1\} \times\{0,1, \ldots, t-1\}$. For $\omega=(u, v) \in A$, the contraction $S_{\omega}\left(x_{1}, x_{2}\right)=\left(\frac{x_{1}}{s}+\frac{u}{s}, \frac{x_{2}}{t}+\frac{v}{t}\right)$ maps the unit square $\mathfrak{R}$ into the rectangle:

$$
\mathfrak{R}_{\omega}=\left[\frac{u}{s}, \frac{u+1}{s}\right] \times\left[\frac{v}{t}, \frac{v+1}{t}\right] .
$$

The (general) Sierpinski carpet K (see $[17,19,29])$ and references therein) is the unique non-empty compact set (see [16]) satisfying

$$
K=\bigcup_{\omega \in A} S_{\omega}(K) .
$$

It is given by:

$$
\begin{aligned}
K & =\left\{x \in \mathfrak{R}:\left(S_{\omega_{1}} \circ \cdots \circ S_{\omega_{n}}\right)^{-1}(x) \in \bigcup_{\omega \in A} \mathfrak{R}_{\omega} \quad \forall \omega=\left(\omega_{1}, \ldots, \omega_{n}\right) \in A^{n}\right\} \\
& =\bigcap_{n \in \mathbb{N}}\left(\bigcup_{\omega \in A^{n}} \mathfrak{R}_{\omega}\right)
\end{aligned}
$$

where

$$
\mathfrak{R}_{\omega}=\left(S_{\omega_{1}} \circ \ldots \circ S_{\omega_{n}}\right)(\mathfrak{R}) \text { for } \omega=\left(\omega_{1}, \ldots, \omega_{n}\right) .
$$

Let $\sigma=\frac{\log s}{\log t}$ and

$$
\left(\alpha_{1}, \alpha_{2}\right)=\left(\frac{2 \sigma}{1+\sigma}, \frac{2}{1+\sigma}\right)=\left(\frac{2 \log s}{\log (s t)}, \frac{2 \log t}{\log s}\right)
$$

By arguments similar to those of [15] pages 118-119, we can prove that $\operatorname{dim}_{\left(\alpha_{1}, \alpha_{2}\right)}(K)=2 \frac{\log a}{\log (s t)}$ where a is the cardinality of $A$, whereas $\operatorname{dim}(K)=\frac{\log \left(\sum_{i=1}^{s} N_{i}^{\sigma}\right)}{\log s}$ where $N_{i}$ is the number of selected rectangles in $A$ from the $i$-th column of the grid (see [15] page 129).

If $s \leq t$ then $\left(\alpha_{\min }, \alpha_{\max }\right)=\left(\alpha_{1}, \alpha_{2}\right)$. By restricting ourselves to the two cases below, we will show that the optimality of (91) may depend on the geometric arrangement of the chosen $\omega$ 's in A. Actually, the left-right side of (91) is optimal in case1 and non optimal in case 2 if $s<t$.

1. Case1: assume that each column of the grid contains at most one $\mathfrak{R}_{\omega}, \omega \in A$. Then $\operatorname{dim} K=\frac{\log a}{\log s}$. Therefore, $\alpha_{1} \operatorname{dim} K=\operatorname{dim}_{\left(\alpha_{1}, \alpha_{2}\right)} K$.

2. Case2: assume that there is only one column containing all the $\mathfrak{R}_{\omega}, \omega \in A$. Then $\operatorname{dim} K=\frac{\sigma \log a}{\log s}$. Therefore, $\frac{\alpha_{2}}{2} \operatorname{dim} K=\operatorname{dim}_{\left(\alpha_{1}, \alpha_{2}\right)} K$.

\section{Fractional Brownian Sheets}

We will apply Theorem 4 for fractional Brownian sheets to show that unlike the Lipschitz scaling function $\eta_{L}(p)$ and the Lipschitz spectum $d(H)$ which are uniform in all directions, the directional scaling function $\eta_{L}(p, e)$ and the directional spectrum $d(H, e)$ are tools that detect directional behaviors. We also provide a directional thermodynamic formalism valid for all fractional Brownian sheets. 
Actually, we will prove that if the corresponding appropriate range $P$ of $p^{\prime}$ s over which one will computes the Legendre transform is given by (9), then $\inf _{p \in P}\left(H p-\eta_{L}(p, e)+1\right)$ provides a common directional Lipschitz scaling based directional thermodynamic formalism.

\subsection{Computation of the Directional Scaling Function}

The fractional Brownian sheet $\left\{B^{\left(H_{1}, \cdots, H_{d}\right)}(y): y=\left(y_{1}, \cdots, y_{d}\right) \in \mathbb{R}^{d}\right\}$ was introduced by Kamont in [67], then redefined by Ayache, Léger, and Pontier in [78] through its harmonizable representation, for any $\left(H_{1}, \cdots, H_{d}\right) \in(0,1)^{d}$

$$
B^{\left(H_{1}, \cdots, H_{d}\right)}(y)=\int_{\mathbb{R}^{d}} \prod_{i \in \mathcal{D}}\left(e^{i y_{i} \xi_{i}}-1\right)\left|\xi_{i}\right|^{-H_{i}-\frac{1}{2}} d \widehat{W}_{\left(\xi_{1}, \cdots, \xi_{d}\right)},
$$

where $\widehat{W}_{\left(\tilde{\xi}_{1}, \cdots, \xi_{d}\right)}$ is the Fourier transform of a Brownian measure $W_{\left(\tilde{\xi}_{1}, \cdots, \xi_{d}\right)}$ on $\mathbb{R}^{d}$.

Fractional Brownian Sheet has stationary rectangular increments and satisfies the following anisotropic scaling relation

$$
\left\{B^{\left(H_{1}, \cdots, H_{d}\right)}\left(a_{1} y_{1}, \cdots, a_{d} y_{d}\right)\right\}_{y \in \mathbb{R}^{d}}=\left\{\left(\prod_{i \in \mathcal{D}} a_{i}^{H_{i}}\right) B^{\left(H_{1}, \cdots, H_{d}\right)}(y)\right\}_{y \in \mathbb{R}^{d}} \quad \text { (same law). }
$$

In [67], Kamont proved that, if $\frac{1}{p}<H_{i}<1$ for all $i \in \mathcal{D}$, then with Probability 1 , the restrictions $B_{I^{d}}^{\left(H_{1}, \cdots, H_{d}\right)}$ of realizations of $B^{\left(H_{1}, \cdots, H_{d}\right)}$ to $I^{d}$ satisfy

$$
B_{I^{d}}^{\left(H_{1}, \cdots, H_{d}\right)} \in \operatorname{Lip}_{p}^{\left(H_{1}, \cdots, H_{d}\right), \frac{1}{2}}\left(I^{d}\right),
$$

and

$$
B_{I^{d}}^{\left(H_{1}, \cdots, H_{d}\right)} \notin \operatorname{lip} p_{p}^{\left(H_{1}, \cdots, H_{d}\right), \frac{1}{2}}\left(I^{d}\right)
$$

Put

$$
H_{\min }=\min \left(H_{1}, \cdots, H_{d}\right) .
$$

We will prove the following result.

Theorem 7. If $\frac{1}{p}<H_{i}<1$ for all $i \in \mathcal{D}$, then with Probability $1, B_{I^{d}}^{\left(H_{1}, \cdots, H_{d}\right)}$ satisfy

$$
\forall i \in \mathcal{D} \quad \eta_{L}\left(p, e_{i}\right)=p H_{i}
$$

and

$$
\eta_{L}(p)=p H_{\min }
$$

Proof. Using the third point in Proposition 6, relation (102) implies that, with Probability 1

$$
B_{I^{d}}^{\left(H_{1}, \cdots, H_{d}\right)} \in \operatorname{Lip}_{p}^{\left(s_{1}, \cdots, s_{d}\right)}\left(I^{d}\right) \quad \forall\left(s_{1}, \cdots, s_{d}\right)<\left(H_{1}, \cdots, H_{d}\right) .
$$

Using the second point in Proposition 6, relation (103) implies that, with Probability 1

$$
B_{I^{d}}^{\left(H_{1}, \cdots, H_{d}\right)} \notin L i p_{p}^{\left(s_{1}, \cdots, s_{d}\right)}\left(I^{d}\right) \quad \forall\left(s_{1}, \cdots, s_{d}\right)>\left(H_{1}, \cdots, H_{d}\right) .
$$

Thanks to the first point in Proposition 5, relation (107) yields the lower bound in (106). 
The optimality of this lower bound cannot be deduced from (108). Nevertheless, the coefficients of $B_{I^{d}}^{\left(H_{1}, \cdots, H_{d}\right)}$ in the tensor product Schauder basis were obtained in [67]; in fact

$$
B_{I^{d}}^{\left(H_{1}, \cdots, H_{d}\right)}=\sum_{\mathbf{j} \in M^{d}} \sum_{\mathbf{k} \in \tilde{N}_{\mathbf{j}}} C_{\mathbf{k}} \Phi_{\mathbf{k}}
$$

where $\left(C_{\mathbf{k}}\right)_{\mathbf{k} \geq \mathbf{0}}$ is a Gaussian sequence, with $E C_{\mathbf{k}}=0$, and the variance given by the formula

$$
E\left|C_{\mathbf{k}}\right|^{2}=\prod_{i \in \mathcal{D}} a_{k_{i}}
$$

where

$$
a_{0}=0, a_{1}=1 \text { and } a_{k_{i}}=\left(2^{-2 H_{i}}-2^{-2}\right) 2^{-2 j_{i} H_{i}} \text { for } k_{i} \in \tilde{N}_{j_{i}} j_{i} \geq 0 .
$$

The above optimality follows immediately from Theorem 4 and arguments similar to those in [92] (p. 236), since both (102), the third result in Proposition 6 and the first result in Proposition 5 imply that $\eta_{L}(p) \geq p H_{\min }>0$. The latest lower bound for $\eta_{L}(p)$ turned out to be equality thanks to (56).

Remark 7. If the unit cube $I^{d}$ is replaced by any arbitrary cube $Q \subset \mathbb{R}^{d}$ then the same arguments applied to the dilated and shifted field $\left\{\rho^{\left|\left(H_{1}, \cdots, H_{d}\right)\right|} B^{\left(H_{1}, \cdots, H_{d}\right)}\left(\rho^{-1} t-c\right): t \in I^{d}\right\},\left(\rho>0, c \in \mathbb{R}^{d}\right)$ give the same result as in Theorem 7.

\subsection{Lipschitz and Directional Spectra and Thermodynamic Formalisms}

We will now compute both Lipschitz spectrum $d(H)$ and directional Lipschitz spectra $d(H, e)$ for $B_{I^{d}}^{\left(H_{1}, \cdots, H_{d}\right)}$. Let us first recall these notions.

Definition 9. Let $f$ be a continuous function on $I^{d}$ (we write $f \in C\left(I^{d}\right)$ ). Let $y \in I^{d}$. Let $0<H<1$. We say that $f \in C^{H}(y)$ if there exists $C>0$, such that:

$$
|f(y+t)-f(y)| \leq C|t|^{H} \quad \forall y+t \in I^{d} .
$$

The pointwise Lipschitz regularity of $f$ at $y$ is:

$$
h(y)=\sup \left\{0<H<1: \quad f \in C^{H}(y)\right\} .
$$

Define the Lipschitz spectrum (respective to upper Lipschitz spectrum) of $f$ as the function $d(H)$ (respective to $D(H)$ ) given by the Hausdorff dimension of the set of points $y$ where $h(y)=H$ (respective to $h(y) \leq H$ ).

We say that $f \in C^{H}\left(I^{d}\right)$, if there exists $C>0$ such that

$$
|f(y+t)-f(y)| \leq C|t|^{H} \quad \forall(y, y+t) \in\left(I^{d}\right)^{2} .
$$

Definition 10. Let $0<H<1$ and $f \in C\left(I^{d}\right)$. Let $e \in S^{d-1}$. Let $y \in I^{d}$.

We say that $f \in C^{H}(y, e)$ if there exists $C>0$ such that

$$
|f(y+t e)-f(y)| \leq C|t|^{H} \quad \forall y+t e \in I^{d} .
$$

The directional pointwise Lipschitz regularity of $f$ at $y$ in direction $e$ is

$$
h(y, e)=\sup \left\{0<H<1: \quad f \in C^{H}(y, e)\right\} .
$$


Define the directional Lipschitz spectrum (respective to directional upper Lipschitz spectrum) of $f$ in direction $e$ as the function $d(H, e)$ (respective to $D(H, e)$ ) given by the Hausdorff dimension of the set of points $y$ where $h(y, e)=H$ (respective to $h(y, e) \leq H)$.

The following theorem provides a common directional thermodynamic formalism for all fractional Brownian sheets.

Theorem 8. With probability 1, both Lipschitz and upper Lipschitz spectra of the restrictions $B_{I^{d}}^{\left(H_{1}, \cdots, H_{d}\right)}$ of realizations of $B^{\left(H_{1}, \cdots, H_{d}\right)}$ are trivial and satisfy the following thermodynamic formalism

$$
\begin{gathered}
d(H)=\left\{\begin{array}{ll}
-\infty & \text { if } H \neq H_{\text {min }} \\
d & \text { if } H=H_{\text {min }}
\end{array}=\inf _{p>1 / H_{\text {min }}}\left(H p-\eta_{L}(p)+d\right) \quad \forall H \leq H_{\text {min }},\right. \\
D(H)=\left\{\begin{array}{ll}
-\infty & \text { if } H<H_{\text {min }} \\
d & \text { if } H \geq H_{\text {min }}
\end{array}=\inf _{p>1 / H_{\text {min }}}\left(H p-\eta_{L}(p)+d\right) \quad \forall H \leq 1 .\right.
\end{gathered}
$$

With probability 1, both directional Lipschitz and directional upper Lipschitz spectra of the restrictions $B_{I^{d}}^{\left(H_{1}, \cdots, H_{d}\right)}$ of realizations of $B^{\left(H_{1}, \cdots, H_{d}\right)}$ are trivial and satisfy the following directional thermodynamic formalism

$$
d\left(H, e_{i}\right)=\left\{\begin{array}{ll}
-\infty & \text { if } H \neq H_{i} \\
d & \text { if } H=H_{i}
\end{array} \quad=\inf _{p>1 / H_{\text {min }}}\left(H p-\eta_{L}\left(p, e_{i}\right)+d\right) \quad \forall H \leq H_{i},\right.
$$

and

$$
D\left(H, e_{i}\right)=\left\{\begin{array}{ll}
-\infty & \text { if } H<H_{i} \\
d & \text { if } H \geq H_{i}
\end{array} \quad=\inf _{p>1 / H_{\text {min }}}\left(H p-\eta_{L}\left(p, e_{i}\right)+d\right) \quad \forall H \leq 1 .\right.
$$

Moreover, Remark 4 holds.

Proof. In [67], we have

$$
B_{I^{d}}^{\left(H_{1}, \cdots, H_{d}\right)} \in C^{H_{\min }}\left(I^{d}\right) .
$$

Let $y=\left(y_{1}, \cdots, y_{d}\right) \in I^{d}$. The unidimensional process $X\left(x_{1}\right)=B_{I^{d}}^{\left(H_{1}, \cdots, H_{d}\right)}\left(x_{1}, y_{2}, \cdots, y_{d}\right)$ is Gaussian, self-similar, with stationary increments and has $H_{1}$ as Hurst index. From the uniqueness of the fractional Brownian motion with Hurst index $H_{1}$, we deduce that $h\left(y, e_{1}\right)=H_{1}$. Similarly, we get $h\left(y, e_{i}\right)=H_{i}$ for all $i \in \mathcal{D}$. Using (121), we deduce that $h(y)=H_{\min }$. The rest of the proof is straightforward.

\section{Sierpinski Cascade Functions}

We will apply Theorem 4 for Sierpinski cascade functions to show that unlike the Lipschitz scaling function $\eta_{L}(p)$ and the Lipschitz spectum $d(H)$ which are uniform in all directions, the directional scaling function $\eta_{L}(p, e)$ and the directional spectrum $d(H, e)$ are tools that detect directional behaviors. We also show that contrary to $\eta_{L}(p, e)$, the directional spectrum $d(H, e)$ depends on the geometric disposition of the chosen contractions for each cascade function. We also provide non common directional Lipschitz scaling based directional thermodynamic formalisms for these examples. These formalisms depend on the geometric disposition of contractions for each cascade function. Nevertheless, all obtained formalisms share the same corresponding appropriate range $P$ of $p^{\prime}$ s over which one will compute the Legendre transform given in (9). Moreover, we show the optimality of Theorem 6 for Sierpinski cascade functions corresponding to a large class of geometric disposition of contractions corresponding to case 1 described in Remark 6. Finally, we modify the notion of the Hausdorff dimension to provide a new common directional Lipschitz scaling based directional thermodynamic formalism for all Sierpinski cascade functions. 
Without any loss of generality, we take $d=2$. A Sierpinski cascade function is a self-similar function adapted to the subdivision $A$ used for the construction of Sierpinski carpet $K$ given in (98). It is written as the superposition of similar anisotropic structures at different scales, reminiscent of some possible modelization of turbulence or cascade models. In [29], we proved that some Sierpinski cascade functions do not satisfy the thermodynamic formalism (5). Put $g(x)=\Lambda\left(x_{1}\right) \Lambda\left(x_{2}\right)$ with $\Lambda(t)=\min (t, 1-t)$ if $t \in[0,1]$ and 0 else. Clearly, $\Lambda(t)=\frac{1}{2} \Phi_{2}(t)$.

The Sierpinski cascade function adapted to the subdivision $A$ satisfies

$$
\forall x \in \mathfrak{R} \quad F(x)=\sum_{\omega \in A} \lambda_{\omega} F\left(S_{\omega}^{-1}(x)\right)+g(x) .
$$

Define

$$
|\lambda|_{\max }=\max _{\omega \in A}\left|\lambda_{\omega}\right|,|\lambda|_{\min }=\min _{\omega \in A}\left|\lambda_{\omega}\right|, H_{\min }=-\frac{\log |\lambda|_{\max }}{\log t} \text { and } H_{\max }=-\frac{\log |\lambda|_{\min }}{\log t} .
$$

The following result was obtained in [29].

Proposition 11. Suppose that $\sum_{\omega \in A}\left|\lambda_{\omega}\right|<s t$, then the series:

$$
F(x)=g(x)+\sum_{n=1}^{\infty} \sum_{\left(\omega_{1}, \ldots, \omega_{n}\right) \in A^{n}} \lambda_{\omega_{1}} \cdots \lambda_{\omega_{n}} g\left(S_{\omega_{n}}^{-1} \cdots S_{\omega_{1}}^{-1}(x)\right) .
$$

is a unique solution in $L^{1}(\mathfrak{R})$ for Equation (122).

If, furthermore, $\frac{1}{t}<|\lambda|_{\max }<1$, then $F \in C^{H_{\min }(\mathfrak{R}) \text { with } 0<H_{\text {min }}<1 .}$

Clearly, if $\omega_{l}=\left(u_{l}, v_{l}\right)$ then

$$
g\left(S_{\omega_{n}}^{-1} \cdots S_{\omega_{1}}^{-1}(x)\right)=\Lambda\left(s^{n} x_{1}-s^{n-1} u_{1}-\cdots-s u_{n-1}-u_{n}\right) \Lambda\left(t^{n} x_{2}-t^{n-1} v_{1}-\cdots-t v_{n-1}-v_{n}\right) .
$$

In [29], we proved that unlike the spectrum $d(H)$, the Lipschitz scaling function $\eta_{L}(p)$ (given in (55)) does not depend on the geometrical arrangement of the chosen $\Re_{\omega}, \omega \in A$, and, so, the multifractal formalism $d(H)=\inf _{p}\left(H p-\eta_{L}(p)+2\right)$ may fail.

\subsection{Computation of the Directional Lipschitz Scaling Function}

Using Theorem 4 and Remark 2, we obtain the following result which shows that, unlike the Lipschitz scaling function $\eta_{L}(p)$ which is uniform in all directions, the directional scaling function $\eta_{L}(p, e)$ is a tool to detect directional behaviors.

Theorem 9. Let $S$ and $T$ be two positive integers. Assume that $s=2^{S}$ and $t=2^{T}$ and $s \leq t$. Assume that $\frac{1}{t}<|\lambda|_{\max }<1$.

Let $1 \leq p<\infty$. Set $\sigma=S / T$ and $\tau(p)=-\frac{\log _{2}\left(\sum_{\omega \in A}\left|\lambda_{\omega}\right|^{p}\right)}{S}$. Let $F$ be the Sierpinski cascade function that corresponds to $A$.

- $\quad$ Suppose that $s<t$ (i.e., $\sigma<1$ ). 
- We have $\frac{t}{s^{p-1}}<\sum_{\omega \in A}\left|\lambda_{\omega}\right|^{p}<s$ is equivalent to $\left(1<\sigma+1+\sigma \tau(p)<p\right.$ and $1<1+\frac{1}{\sigma}+$ $\tau(p)<p)$. In that case,

$$
\eta_{L}\left(p, e_{1}\right)=1+\frac{1}{\sigma}+\tau(p), \eta_{L}\left(p, e_{2}\right)=\sigma+1+\sigma \tau(p) .
$$

and

$$
\forall e \neq \pm e_{1} \quad \eta_{L}(p, e)=\sigma+1+\sigma \tau(p)=\eta_{L}(p) .
$$

- We have $\frac{s}{t^{p-1}}<\sum_{\omega \in A}\left|\lambda_{\omega}\right|^{p}<s$ and $\sum_{\omega \in A}\left|\lambda_{\omega}\right|^{p} \leq \frac{t}{s^{p-1}}$ is equivalent to $(1<\sigma+1+\sigma \tau(p)<p$ and $\left.1+\frac{1}{\sigma}+\tau(p) \geq p\right)$. In that case

$$
\eta_{L}\left(p, e_{1}\right)=p, \eta_{L}\left(p, e_{2}\right)=\sigma+1+\sigma \tau(p)
$$

and

$$
\forall e \neq \pm e_{1} \quad \eta_{L}(p, e)=\sigma+1+\sigma \tau(p)=\eta_{L}(p) .
$$

- In the case $\sum_{\omega \in A}\left|\lambda_{\omega}\right|^{p} \leq \frac{s}{t^{p-1}}$, we have $\eta_{L}\left(p, e_{1}\right)=\eta_{L}\left(p, e_{2}\right)=p$.

- $\quad$ Suppose that $s=t$ (i.e., $\sigma=1$ ).

- We have $\frac{s}{s^{p-1}}<\sum_{\omega \in A}\left|\lambda_{\omega}\right|^{p}<s$ is equivalent to $1<2+\tau(p)<p$. In that case,

$$
\eta_{L}\left(p, e_{1}\right)=2+\tau(p) \text { and } \eta_{L}\left(p, e_{2}\right)=2+\tau(p) .
$$

- In the case where $\sum_{\omega \in A}\left|\lambda_{\omega}\right|^{p} \leq \frac{s}{s^{p-1}}$, we have $2+\tau(p) \geq p$, therefore,

$$
\eta_{L}\left(p, e_{1}\right)=\eta_{L}\left(p, e_{2}\right)=p
$$

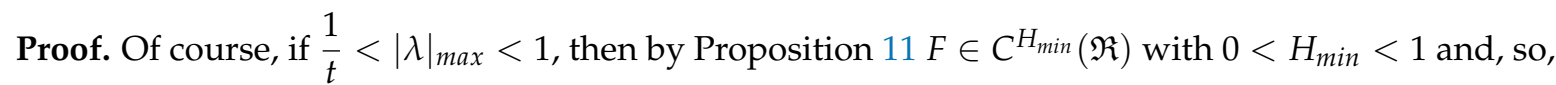
$\eta_{L}(p)>0$.

For $\mathbf{j}=\left(j_{1}, j_{2}\right)=(n S, n T)$, we have

$$
\tau_{\mathbf{j}, p}(f)=2^{-n(S+T) / p}\left(\sum_{\omega \in A}\left|\lambda_{\omega}\right|^{p}\right)^{n / p}
$$

It follows that

$$
\frac{\log \tau_{\mathbf{j}, p}(f)}{\log \left(2^{-j_{1}}\right)}=\frac{1}{p}\left(1+\frac{T}{S}-\frac{1}{S} \log _{2}\left(\sum_{\omega \in A}\left|\lambda_{\omega}\right|^{p}\right)\right)=\frac{1}{p}\left(1+\frac{1}{\sigma}+\tau(p)\right) .
$$

and

$$
\frac{\log \tau_{\mathbf{j}, p}(f)}{\log \left(2^{-j_{2}}\right)}=\sigma \frac{\log \tau_{\mathbf{j}, p}(f)}{\log \left(2^{-j_{1}}\right)}=\frac{1}{p}(\sigma+1+\sigma \tau(p))
$$

In order to apply Theorem 4 , we need that $\liminf _{|\mathbf{j}| \rightarrow \infty} \frac{\log \tau_{\mathbf{j}, p}(f)}{\log \left(2^{-j_{i}}\right)}>1 / p$ for every $i=1$, 2. Clearly,

$$
\liminf _{|\mathbf{j}| \rightarrow \infty} \frac{\log \tau_{\mathbf{j}, p}(f)}{\log \left(2^{-j_{2}}\right)}>1 / p \Leftrightarrow \sigma+1+\sigma \tau(p)>1 \Leftrightarrow \tau(p)>-1 \Leftrightarrow \sum_{\omega \in A}\left|\lambda_{\omega}\right|^{p}<s,
$$




$$
\begin{gathered}
\liminf _{|\mathbf{j}| \rightarrow \infty} \frac{\log \tau_{\mathbf{j}, p}(f)}{\log \left(2^{-j_{2}}\right)}<1 \Leftrightarrow \sigma+1+\sigma \tau(p)<p \Leftrightarrow \frac{s}{t^{p-1}}<\sum_{\omega \in A}\left|\lambda_{\omega}\right|^{p}, \\
\liminf _{|\mathbf{j}| \rightarrow \infty} \frac{\log \tau_{\mathbf{j}, p}(f)}{\log \left(2^{-j_{1}}\right)}>1 / p \Leftrightarrow 1+\frac{1}{\sigma}+\tau(p)>1 \Leftrightarrow \tau(p)>-1 / \sigma \Leftrightarrow \sum_{\omega \in A}\left|\lambda_{\omega}\right|^{p}<t
\end{gathered}
$$

and

$$
\liminf _{|\mathbf{j}| \rightarrow \infty} \frac{\log \tau_{\mathbf{j}, p}(f)}{\log \left(2^{-j_{1}}\right)}<1 \Leftrightarrow 1+\frac{1}{\sigma}+\tau(p)<p \Leftrightarrow \frac{t}{s^{p-1}}<\sum_{\omega \in A}\left|\lambda_{\omega}\right|^{p}
$$

Clearly, since $s \leq t$ then $\frac{s}{t^{p-1}} \leq \frac{t}{s^{p-1}}$.

- $\quad$ Suppose that $s<t$ (i.e., $\sigma<1$ ).

- If $\frac{t}{s^{p-1}}<\sum_{\omega \in A}\left|\lambda_{\omega}\right|^{p}<s$ then $p>1 / \sigma, \eta_{L}\left(p, e_{1}\right)=1+\frac{1}{\sigma}+\tau(p)$ and $\eta_{L}\left(p, e_{2}\right)=\sigma+1+$ $\sigma \tau(p)$. So, using Remark 2, we deduce that $\eta_{L}(p, e)=\sigma+1+\sigma \tau(p)=\eta_{L}(p)$ for all $e \neq \pm e_{1}$.

- If $\frac{s}{t^{p-1}}<\sum_{\omega \in A}\left|\lambda_{\omega}\right|^{p}<s$ and $\sum_{\omega \in A}\left|\lambda_{\omega}\right|^{p} \leq \frac{s^{p-1}}{s^{p-1}}$ then $\eta_{L}\left(p, e_{1}\right)=p$ and $\eta_{L}\left(p, e_{2}\right)=\sigma+1+$ $\sigma \tau(p)$. So, using Remark 2, we deduce that $\eta_{L}(p, e)=\sigma+1+\sigma \tau(p)=\eta_{L}(p)$ for all $e \neq \pm e_{1}$. Note that $s \leq \frac{t}{s^{p-1}}$ iff $p \leq 1 / \sigma$.

- If $\sum_{\omega \in A}\left|\lambda_{\omega}\right|^{p} \leq \frac{s}{t^{p-1}}$ then $\eta_{L}\left(p, e_{1}\right)=\eta_{L}\left(p, e_{2}\right)=p$.

- $\quad$ Suppose that $s=t$.

- We have $\frac{s}{s^{p-1}}<\sum_{\omega \in A}\left|\lambda_{\omega}\right|^{p}<s$ is equivalent to $1<2+\tau(p)<p$. In that case

$$
\eta_{L}\left(p, e_{1}\right)=2+\tau(p) \text { and } \eta_{L}\left(p, e_{2}\right)=2+\tau(p) .
$$

- In the case where $\sum_{\omega \in A}\left|\lambda_{\omega}\right|^{p} \leq \frac{s}{s^{p-1}}$, we have $2+\tau(p) \geq p$, therefore,

$$
\eta_{L}\left(p, e_{1}\right)=\eta_{L}\left(p, e_{2}\right)=p
$$

Remark 8. Theorem 9 improves previous results in [29] without any assumptions on the choice of $\omega \in A$ and the positivity of the corresponding $\lambda_{\omega}$. Recall that in [29], we were interested in the computation of $\eta_{L}(p)$ by the increments method.

\subsection{Directional Pointwise Lipschitz Regularity}

We will now compute the pointwise directional Lipschitz regularity of the Sierpinski function. In [29], we were interested in the computation of the pointwise Lipschitz regularity. Let us recall the obtained results. Consider the "separated open set condition":

$$
\forall\left(\omega, \omega^{\prime}\right) \in A^{2} \quad \omega \neq \omega^{\prime} \Rightarrow \Re_{\omega} \cap \Re_{\omega^{\prime}}=\varnothing .
$$

Recall that $K$ is the Sierpinski carpet (98).

$$
\forall x \notin K \quad h(x)=1 .
$$

Define for $x \in K, \omega=\omega(x)=\left(\omega_{1}, \omega_{2}, \ldots, \omega_{n}, \ldots\right) \in A^{\mathbb{N}}$ by $\omega_{l}=\left(u_{l}, v_{l}\right) \in A$ with $x=\left(\sum_{l=1}^{\infty} \frac{u_{l}}{s^{l}}, \sum_{l=1}^{\infty} \frac{v_{l}}{t^{l}}\right)$. 
Denote by

$$
\begin{gathered}
\omega(n, x)=\left(\omega_{1}, \ldots, \omega_{n}\right), \lambda_{\omega(n, x)}=\lambda_{\omega_{1}} \cdots \lambda_{\omega_{n}} \\
a_{t}(x)=\liminf _{n \mapsto \infty} \frac{\log \left|\lambda_{\omega(n, x)}\right|}{\log t^{-n}} \text { and } a_{s}(x)=\liminf _{n \mapsto \infty} \frac{\log \left|\lambda_{\omega(n, x)}\right|}{\log \mathcal{s}^{-n}} .
\end{gathered}
$$

In Proposition 3 in [29], using increments method for the Sierpinski cascade function, we proved that $h(x) \geq a_{t}(x)$ under assumptions (124), $a_{t}(x)<1$ and

$$
\forall \omega \in A, \quad \mathfrak{R}_{\omega} \subset[1 / s, 1-1 / s] \times[1 / t, 1-1 / t] .
$$

This yields

$$
h\left(x, e_{2}\right) \geq a_{t}(x)
$$

Similar arguments allow us to obtain

$$
h\left(x, e_{1}\right) \geq a_{s}(x) \text { if } a_{s}(x)<1 .
$$

In Proposition 4 in [29], using increments method, we proved that $h(x) \leq a_{t}(x)$ under assumptions (124), $a_{t}(x) \leq 1$

$$
0<\lambda_{\omega}<1 \quad \forall \omega \in A,
$$

and either:

$$
\forall \omega \in A, \quad \mathfrak{R}_{\omega} \subset[1 / s, 1-1 / s] \times[1 / t, 1 / 2],
$$

or

$$
\forall \omega \in A, \quad \mathfrak{R}_{\omega} \subset[1 / s, 1-1 / s] \times[1 / 2,1-1 / t] .
$$

Actually, we proved that $h\left(x, e_{2}\right) \leq a_{t}(x)$. We deduce that:

$$
h(x)=h\left(x, e_{2}\right)=a_{t}(x) .
$$

We will improve result (132) and obtain a similar result for $h\left(x, e_{1}\right)$ without adding assumption (130) nor (131). For that we will use Theorem 4 obtained in [58], in which we characterized directional pointwise Lipschitz regularity in terms of decay conditions for the coefficients $C_{\mathbf{k}}(f)$ (given in (76)) of the expansion of $f$ in the basis of tensor products of Schauder functions. Let us recall this result for $d=2$; if $k_{i} \in \tilde{N}_{j_{i}}$, with $k_{i} \geq 2$ then $\phi_{k_{i}}$ has support $\left[\left(n_{i}-1\right) 2^{-j_{i}}, n_{i} 2^{-j_{i}}\right.$. It follows that for $j_{i} \in M$ with $j_{i} \geq 0$ and $x \in I^{2}$, there exists a unique value of $k_{i}\left(x_{i}\right)$ for which $x_{i} \in\left[\left(n_{i}-1\right) 2^{-j_{i}}, n_{i} 2^{-j_{i}}\right)$. We keep the notation $\left.k_{i}(x)\right)$ even if $j_{i} \in\{-2,-1\}$.

Proposition 12. Let $x \in I^{2}$ and $f \in C\left(I^{2}\right)$. Set

$$
\rho\left(x, e_{1}\right)=\liminf _{j_{1} \rightarrow \infty} \inf _{k_{1} \in \tilde{N}_{j_{1}}} \inf _{j_{2} \in M} \frac{\log \left(\left|C_{\left(k_{1}, k_{2}\left(x_{2}\right)\right)}(f)\right| \phi_{k_{2}\left(x_{2}\right)}\left(x_{2}\right)\right)}{\log \left(2^{-j_{1}}+\left|n_{1} 2^{-j_{1}}-x_{1}\right|\right)}
$$

and

$$
\rho\left(x, e_{2}\right)=\liminf _{j_{2} \rightarrow \infty} \inf _{k_{2} \in \tilde{N}_{j_{2}}} \inf _{j_{1} \in M} \frac{\log \left(\left|C_{\left(k_{1}\left(x_{1}\right), k_{2}\right)}(f)\right| \phi_{k_{1}\left(x_{1}\right)}\left(x_{1}\right)\right)}{\log \left(2^{-j_{2}}+\left|n_{2} 2^{-j_{2}}-x_{2}\right|\right)}
$$

Assume that $f$ is uniformly Lipschitz regular on $I^{2}$ in direction $e_{1}$ in the sense that there exists $\delta>0$ and $C>0$, such that $\left|f\left(x+t e_{1}\right)-f(x)\right| \leq C|t|^{\delta}$ for all $x$ and $x+t e_{1} \in I^{2}$.

If:

$$
\forall \mathbf{k} \quad C_{\left(k_{1}, k_{2}\left(x_{2}\right)\right)}(f) \geq 0,
$$


then:

$$
h\left(x, e_{1}\right)=\min \left(1, \rho\left(x, e_{1}\right)\right) .
$$

Assume that $f$ is uniformly Lipschitz regular on $I^{2}$ in direction $e_{2}$.

If:

$$
\forall \mathbf{k} \quad C_{\left(k_{1}\left(x_{1}\right), k_{2}\right)}(f) \geq 0,
$$

then:

$$
h\left(x, e_{2}\right)=\min \left(1, \rho\left(x, e_{2}\right)\right) .
$$

Theorem 10. Assume $\lambda_{\max }>1 / t,(124),(126),(129)$ and (142). Then, for the Sierpinski cascade function:

$$
h\left(x, e_{1}\right)= \begin{cases}1 & \text { if } x \notin K \\ a_{s}(x) & \text { if } x \in K\end{cases}
$$

and

$$
h\left(x, e_{2}\right)=h(x)= \begin{cases}1 & \text { if } x \notin K \\ a_{t}(x) & \text { if } x \in K .\end{cases}
$$

Proof. Results for $x \notin K$ follow from (125).

Assumption (129) yields both (133) and (135). On the other hand, by assumption $\lambda_{\max }>1 / t$, Proposition 11 implies that $F \in C^{H_{\min }(\mathfrak{R})}$ with $0<H_{\min }<1$. This implies that $F$ is uniformly Lipschitz regular on $I^{2}$ in any direction. By Proposition 12 , for $x \in K$ and $i=1,2$ :

$$
h\left(x, e_{i}\right)=\min \left(1, \rho\left(x, e_{i}\right)\right) .
$$

Of course,

$$
\rho\left(x, e_{1}\right) \leq \liminf _{j_{1} \rightarrow \infty} \inf _{k_{1}\left(x_{1}\right) \in \tilde{N}_{j_{1}}} \inf _{j_{2} \in M} \frac{\log \left(C_{\left(k_{1}\left(x_{1}\right), k_{2}\left(x_{2}\right)\right)}(f) \phi_{k_{2}\left(x_{2}\right)}\left(x_{2}\right)\right)}{\log \left(2^{-j_{1}}+\left|n_{1} 2^{-j_{1}}-x_{1}\right|\right)} .
$$

Assumption (126) yields:

$$
\rho\left(x, e_{1}\right) \leq a_{s}(x)
$$

because from the definition of $\left(k_{1}\left(x_{1}\right), k_{2}\left(x_{2}\right)\right)$, we have $\left|n_{1} 2^{-j_{1}}-x_{1}\right| \leq 2^{-j_{1}}$ and if $x_{2}=\sum_{l=1}^{\infty} \frac{v_{l}}{t^{l}}$ then thanks to assumption (126):

$$
\phi_{k_{2}\left(x_{2}\right)}\left(x_{2}\right)=2 \Lambda\left(t^{n} x_{2}-t^{n-1} v_{1}-\cdots-t v_{n-1}-v_{n}\right)=2 \Lambda\left(\sum_{l=1}^{\infty} \frac{v_{n+l}}{t^{l}}\right) \geq \frac{1}{t} .
$$

Similarly, assumption (126) yields:

$$
\rho\left(x, e_{2}\right) \leq a_{t}(x) .
$$

Properties (139), (140), and (141) make equalities in results (127) and (128) (under assumptions (124) and (126)).

\subsection{Directional Pointwise Lipschitz Spectrum and Directional Thermodynamic Formalisms}

We will now compute the directional Lipschitz spectrum of the Sierpinski function and provide directional thermodynamic formalisms. We will see that, unlike the directional Lipschitz scaling function $\eta_{L}(p, e)$, the directional spectrum $d(H, e)$ (and, so, the directional thermodynamic formalisms) may depend on the geometric arrangement of the chosen $R_{\omega}$. Actually, in [29], we proved a similar property for the Lipschitz scaling function $\eta_{L}(p)$ and the Lipschitz spectrum $d(H)$. Nevertheless, we will show that unlike the Lipschitz spectrum $d(H)$ which is uniform in all directions, the directional Lipschitz spectrum $d(H, e)$ may depend on $e$ and consequently is a tool to detect directional behaviors. 
Assume that if column $u$ of the grid contains points of $K$, then the two adjacent columns do not, i.e.,

$$
\text { if } \omega=(u, v) \in A \text { then }(u \pm 1, v) \notin A .
$$

The following theorem holds. It provides directional thermodynamic formalisms valid for the Sierpinski function.

Theorem 11. Assume $\lambda_{\max }>1 / t,(124),(126),(129)$, and (142). Let $F$ be the corresponding Sierpinski cascade function.

The set $P$ given in (9) is:

$$
P=\left\{p \geq 1: \frac{t}{s^{p-1}}<\sum_{\omega \in A}\left|\lambda_{\omega}\right|^{p}<s\right\}
$$

Put:

$$
\tau^{\prime}(P)=\left\{\tau^{\prime}(p): p \in P\right\} \text { and } \sigma \tau^{\prime}(P)=\left\{\sigma \tau^{\prime}(p): p \in P\right\} .
$$

1. Case 1: Assume that each column of the grid contains at most one $\mathfrak{R}_{\omega}, \omega \in A$. Then:

$$
d\left(H, e_{2}\right)=d(H)= \begin{cases}-\infty & \text { if } H<H_{\text {min }} \\ \inf _{q}\left(q \sigma^{-1} H-\tau(q)\right) & \text { if } H \in\left[H_{\text {min }}, \min \left(1, H_{\text {max }}\right)\right]\end{cases}
$$

and

$$
d\left(H, e_{1}\right)=d\left(H \sigma, e_{2}\right)= \begin{cases}-\infty & \text { if } H \sigma<H_{\min } \\ \inf _{q}(q H-\tau(q)) & \text { if } H \sigma \in\left[H_{\min }, \min \left(1, H_{\max }\right)\right]\end{cases}
$$

The following directional thermodynamic formalisms hold:

$$
\forall H \in \tau^{\prime}(P) \cap\left(-\infty, \frac{1}{\sigma} \min \left(1, H_{\max }\right)\right] \quad d\left(H, e_{1}\right)=\inf _{p \in P}\left(p H-\eta_{L}\left(p, e_{1}\right)+1+\frac{1}{\sigma}\right)
$$

and

$$
\forall H \in \sigma \tau^{\prime}(P) \cap\left(-\infty, \min \left(1, H_{\max }\right)\right] \quad d\left(H, e_{2}\right)=\frac{1}{\sigma} \inf _{p \in P}\left(p H-\eta_{L}\left(p, e_{2}\right)+1+\sigma\right) .
$$

2. Case 2: Assume that there is only one column containing all the $\mathfrak{R}_{\omega}, \omega \in A$. Then:

$$
d\left(H, e_{2}\right)=d(H)= \begin{cases}-\infty & \text { if } H<H_{\text {min }} \\ \inf _{q}(q H-\sigma \tau(q)) & \text { if } H \in\left[H_{\text {min }}, \min \left(1, H_{\text {max }}\right)\right]\end{cases}
$$

and

$$
d\left(H, e_{1}\right)=d\left(H \sigma, e_{2}\right)= \begin{cases}-\infty & \text { if } H \sigma<H_{\text {min }} \\ \sigma \inf _{q}(q H-\tau(q)) & \text { if } H \sigma \in\left[H_{\min }, \min \left(1, H_{\text {max }}\right)\right] .\end{cases}
$$

The following directional thermodynamic formalisms hold:

$$
\forall H \in \tau^{\prime}(P) \cap\left(-\infty, \frac{1}{\sigma} \min \left(1, H_{\text {max }}\right)\right] \quad d\left(H, e_{1}\right)=\sigma \inf _{p \in P}\left(p H-\eta_{L}\left(p, e_{1}\right)+1+\frac{1}{\sigma}\right)
$$

and

$$
\forall H \in \sigma \tau^{\prime}(P) \cap\left(-\infty, \min \left(1, H_{\max }\right)\right] \quad d\left(H, e_{2}\right)=\inf _{p \in P}\left(p H-\eta_{L}\left(p, e_{2}\right)+1+\sigma\right) .
$$


Proof. Relation (143) is direct consequence of Theorem 9.

Since $s \leq t$ then (132) yields $d(H)=d\left(H, e_{2}\right)$, therefore, results of Proposition 5 in [29] (respective to Proposition 6 in [29]) remain valid for $d(H)$ replaced by $d\left(H, e_{2}\right)$ with the same conditions except for (130) or (131) which can be replaced by the weaker condition (126) (see the previous section). Moreover, results (146) and (150) follow from the fact that $a_{s}(t)=\frac{1}{\sigma} a_{t}(x)$. The above thermodynamic formalisms follow directly from Theorem 9 .

Remark 9. When $s=t$, the above thermodynamic formalisms coincide with the classical formalism.

\subsection{Optimality of Theorem 6 in Case 1}

We will prove that Theorem 6 is optimal in case1, in the sense that the upper bound (96) becomes equality.

Theorem 12. Let $\mathcal{B}$ and $\Omega$ be as in Theorem 1. Assume $\lambda_{\max }>1 / t,(124),(126),(129)$ and (142). Let $F$ be the corresponding Sierpinski cascade function.

Assume that each column of the grid contains at most one $\mathfrak{R}_{\omega}$. Then:

$$
\forall H \leq 1 \quad D(H, e)=\inf _{\alpha \in \Omega} \frac{1}{\alpha_{\min }} \inf _{p \geq p_{\alpha}}\left(\alpha_{1} H p-\eta_{\alpha}(p)+d\right) .
$$

Proof. If $\left(\alpha_{1}, \alpha_{2}\right)$ is given by (99), then $\alpha_{\min }=\alpha_{1}, p_{\left(\alpha_{1}, \alpha_{2}\right)}$ given in (95) satisfies $\tau\left(p_{\left(\alpha_{1}, \alpha_{2}\right)}\right)=0$, and

$$
\forall i \in\{1,2\} \quad \eta_{L}\left(p, e_{i}\right)=\frac{\eta_{\left(\alpha_{1}, \alpha_{2}\right)}(p)}{\alpha_{i}} .
$$

Relations (147) and (148) (in case 1), respectively, can be rewritten as:

$$
\forall H \in \tau^{\prime}(P) \cap\left(-\infty, \frac{1}{\sigma} \min \left(1, H_{\max }\right)\right] \quad d\left(H, e_{1}\right)=\frac{1}{\alpha_{\min }} \inf _{p \in P}\left(\alpha_{1} H p-\eta_{\left(\alpha_{1}, \alpha_{2}\right)}(p)+2\right)
$$

and

$$
\forall H \in \sigma \tau^{\prime}(P) \cap\left(-\infty, \min \left(1, H_{\max }\right)\right] \quad d\left(H, e_{2}\right)=\frac{1}{\alpha_{\min }} \inf _{p \in P}\left(\alpha_{2} H p-\eta_{\left(\alpha_{1}, \alpha_{2}\right)}(p)+2\right) .
$$

Since:

$$
\left\{p \geq p_{\left(\alpha_{1}, \alpha_{2}\right)}\right\} \subset P,
$$

then the previous upper bounds become equalities and (153) holds.

\subsection{Directional Thermodynamic Formalisms Independent on the Choice of $A$}

We will modify the notion of the Hausdorff dimension to provide a new directional thermodynamic formalism independent on the choice of $A$. For any $n \geq 1, \mathcal{T}_{n}:=\left\{\mathfrak{R} \omega ; \omega \in A^{n}\right\}$ is a partition of $K$. Let $\mathcal{T}=\bigcup_{n \geq 1} \mathcal{T}_{n}$. Define $\operatorname{dim}_{\mathcal{T}}$ in a similar way to the Hausdorff dimension but by considering only coverings by elements of $\mathcal{T}$. Note that such 'restriction' to the elements of dynamics was done by many authors (see [11-14,20,30]). Of course, the diameter $\left|\mathfrak{R}_{\omega}\right|$ for $\omega \in A^{n}$ can be replaced by $s^{-n}$ (because it is equivalent to $s^{-n}$ ). Define the modified directional Lipschitz $\mathcal{T}$ spectrum (respective to directional upper Lipschitz $\mathcal{T}$ spectrum) of $f$ in direction $e$ as the function $d_{\mathcal{T}}(H, e)$ (respective to $D_{\mathcal{T}}(H, e)$ ) given by the $\operatorname{dim}_{\mathcal{T}}$ of the set of points $y$ where $h(y, e)=H$ (respective to $h(y, e) \leq H)$. 
Theorem 13. Assume $\lambda_{\max }>1 / t,(124),(126),(129)$ and (142). Let $F$ be the corresponding Sierpinski cascade function. Let $P$ as in (143). Then the following directional thermodynamic formalisms hold:

$$
\forall H \in \tau^{\prime}(P) \cap\left(-\infty, \min \left(1, H_{\max }\right)\right] \quad d_{\mathcal{T}}\left(H, e_{1}\right)=\inf _{p \in P}\left(p H-\eta_{L}\left(p, e_{1}\right)+1+\frac{1}{\sigma}\right)
$$

and

$$
\forall H \in \sigma \tau^{\prime}(P) \cap\left(-\infty, \min \left(1, H_{\max }\right)\right] \quad d_{\mathcal{T}}\left(H, e_{2}\right)=\frac{1}{\sigma} \inf _{p \in P}\left(p H-\eta_{L}\left(p, e_{2}\right)+1+\sigma\right) .
$$

Proof. Set $P_{\omega}(q)=\lambda_{\omega}^{q} s^{\tau(q)}$ and let $\mu_{q}$ be a probability measure on $K$ such that:

$$
\forall\left(\omega_{1}, \ldots, \omega_{n}\right) \in A^{n} \quad \mu_{q}\left(\Re_{\omega_{1}, \ldots, \omega_{n}}\right)=P_{\omega_{1}}(q) \ldots P_{\omega_{n}}(q) .
$$

Since:

$$
\mu_{q}\left(\Re_{\omega_{1}, \ldots, \omega_{n}, \omega_{1}^{\prime}, \ldots, \omega_{m}^{\prime}}\right)=\mu_{q}\left(\Re_{\omega_{1}, \ldots, \omega_{n}}\right) \mu_{q}\left(\Re_{\omega_{1}^{\prime}, \ldots, \omega_{m}^{\prime}}\right),
$$

then, as in $[25,30]$, we can concentrate a Gibbs measure $v_{p}$ on $E_{F}^{\varphi^{\prime}(p)}$, i.e.,

$$
\forall \omega \quad v_{p}\left(\Re_{\omega}\right) \simeq\left(\mu\left(\Re_{\omega}\right)\right)^{p}\left|\mathfrak{R}_{\omega}\right|^{-\varphi(p)}
$$

where $\varphi$ is defined as in [13], and, thus, we obtain:

$$
d_{\mathcal{T}}\left(H, e_{1}\right)=\inf _{q}(H q-\tau(q))
$$

and

$$
d_{\mathcal{T}}\left(H, e_{2}\right)=\inf _{q}\left(\frac{H}{\sigma} q-\tau(q)\right)=\frac{1}{\sigma} \inf _{q}(H q-\sigma \tau(q)) .
$$

Therefore, (154) and (155) hold.

Remark 10. The new formalism shows also that if $D(H, e)$ in Theorem 6 is replaced by $D_{\mathcal{T}}(H, e)$ then we get optimality independently on the choice of $A$.

\section{Motivation of the Anisotropic Cascade Model on the Physics Side}

In all realistic flows in turbulence, there always exists some anisotropy at all scales (for example, see [93] and references therein); the statistical properties of the velocity field are effected by the geometry of the boundaries or the driving mechanism, which are never rotationally invariant [94]. For example, all geophysical flows are subject to the rotation of the globe, which introduces anisotropy via the Coriolis forces [95]. There is also a whole literature on anisotropy in turbulence created by vortex stretching (for example, see [96] and references therein). It has been a grand challenge in the mathematical fluid mechanics community to try to explain/quantify the process of anisotropic dissipation in turbulent flows directly from the mathematical model the 3D Navier-Stokes equations NSE. In [97], Constantin derived a singular integral representation of the stretching factor in the evolution of the vorticity magnitude featuring a geometric kernel that is depleted by local coherence of the vorticity direction. In [98], Ran showed that there are dynamical systems that are much simpler than the NSE but that can still have turbulent states and for which many concepts developed in the theory of dynamical systems can be successfully applied.

Clearly, cascade models introduced to model turbulence (the cascade picture of turbulent flows takes its origin from Richardson in 1922) should be able to take into account anisotropy. If we want to be able to make model selection with, we must use an anisotropic multifractal formalism as the equality in (94). 
Author Contributions: All authors contributed to this article.

Funding: Mourad Ben Slimane and Borhen Halouani extend their appreciation to the Deanship of Scientific Research at King Saud University for funding this work through research group No (RG-1435-063).

Acknowledgments: Mourad Ben Slimane would like to thank Marianne Clausel, Stéphane Jaffard and Béatrice Vedel for stimulating discussions. We are very grateful to the referees for their comments and detailed suggestions which helped us to improve the manuscript.

Conflicts of Interest: The authors declare no conflict of interest.

\section{References}

1. Benzi, R.; Paladin, G.; Parisi, G.; Vulpani, A. On the multifractal nature of turbulence and chotic systems. J. Phys. A 1984, 17, 3521-3531. [CrossRef]

2. Mandelbrot, B. Intermittent turbulence in selfsimilar cascades: Divergence of high moments and dimension of the carrier. J. Fluid Mech. 1974, 62, 331-358. [CrossRef]

3. Eckmann, B.; Ruelle, D. Ergodic theory of chaos and strange attractors. Rev. Mod. Phys. 1985, 57, 617-656. [CrossRef]

4. Halsey, T.-C.; Jensen, M.-H.; Kadaroff, L.-P.; Procaccia, I.; Shraiman, B.-I. Fractal measures and their singularities: The charaterization of strange sets. Phys. Rev. A 1986, 33, 1141-1151. [CrossRef]

5. Kolmogorov, A.N. Energy dissipation in locally isotropic turbulence. Dokl. Akad. Nauk. 1941, 32, 16-18. [CrossRef]

6. Kolmogorov, A.N. A refinement of previous hypotheses concerning the local structure of turbulence in a viscous incompressible fluid at high Reynolds number. J. Fluid Mech. 1962, 12, 8285. [CrossRef]

7. Oboukhov, A.M. Some specific features of atmospheric tubulence. J. Fluid Mech. 1962, 12, 7781. [CrossRef]

8. Mandelbrot, B. Les Objets Fractals: Forme, Hasard et Dimension; Flammarian: Paris, France, 1975.

9. Mandelbrot, B. The Fractal Geometry of Nature; W. H. Freeman: New York, NY, USA, 1982.

10. Frisch, U.; Parisi, G. Fully developped turbulence and intermittency. In Proceedings of the International Summer School in Physics; Fermi, E., Ed.; North-Holland: Amsterdam, The Netherlands, 1985; pp. 84-88.

11. Ben Nasr, F. Multifractal analysis of measures. C. R. Acad. Sci. Paris Sér. I Math. 1994, 319, 807-810.

12. Ben Nasr, F.; Bhouri, I.; Heurtaux, Y. A necessary condition and sufficientn condition for a valid multifractal formalism. Math. Adv. Math. 2002, 165, 264-284. [CrossRef]

13. Brown, G.; Michon, G.; Peyrière, J. On the multifractal analysis of measures. J. Statist. Phys. 1992, 66, 775-790. [CrossRef]

14. Collet, P.; Lebowitz, J.; Porzio, A. The dimension specrum of some dynamical systems. J. Statist. Phys. 1987, 47, 609-644. [CrossRef]

15. Falconer, K.-J. Fractal Geometry: Mathematical Foundations and Applications; John Wiley and Sons: Toronto, ON, Canada, 1990.

16. Hutchinson, J. Fractals and self-similarity. Indiana Univ. Math. J. 1981, 30, 713-747. [CrossRef]

17. King, J. The singularity spectrum for general Sierpinski carpets. Adv. Math. 1995, 116, 1-11. [CrossRef]

18. Olsen, L. A multifractal formalism. Adv. Math. 1995, 116, 92-195. [CrossRef]

19. Olsen, L. Self-affine multifractal Sierpinski sponges in $R^{d}$. Pac. J. Math. 1998, 183, 143-199. [CrossRef]

20. Rand, D.A. The singularity spectrum $f(\alpha)$ for cookie-cutters. Ergodic Theory Dyn. Syst. 1989, 9, 527-541. [CrossRef]

21. Daubechies, I.; Lagarias, J.-C. On the thermodynamic formalism for functions. Rev. Math. Phys. 1994, 6, 1033-1070. [CrossRef]

22. Arneodo, A.; Bacry, E.; Muzy, J.-F. Singularity spectrum of fractal signals from wavelet analysis: Exact results. J. Statist. Phys. 1993, 70, 635-674.

23. Arneodo, A.; Bacry, E.; Muzy, J.-F. The thermodynamics of fractals revisited with wavelets. Physica A 1995, 213, 232-275. [CrossRef]

24. Muzy, J.-F.; Arneodo, A.; Bacry, E. A multifractal formalism revisited with wavelets. Internat. J. Bifur. Chaos Appl. Sci. Engrg. 1994, 4, 245. [CrossRef]

25. Jaffard, S. Multifractal formalism for functions. Part 1: Results valid for all functions and Part 2: Selfsimilar functions. SIAM J. Math. Anal. 1997, 28, 944-998. [CrossRef]

26. Ben Abid, M.; Seuret, S. Hölder regularity of $\mu$-similar functions. Const. Approx. 2010, 31, 69-93. [CrossRef] 
27. Ben Slimane, M. Etude du Formalisme Multifractal pour les Fonctions. Ph.D. Thesis, Ecole Nationale des Ponts et Chaussées, Paris, France, 1996.

28. Ben Slimane, M. Formalisme Multifractal pour quelques généralisations des fonctions autosimilaires. C. $R$. Acad. Sci. Paris Sér. I Math. 1997, 324, 981-986. [CrossRef]

29. Ben Slimane, M. Multifractal formalism and anisotropic selfsimilar functions. Math. Proc. Camb. Philos. Soc. 1998, 124, 329-363. [CrossRef]

30. Ben Slimane, M. Multifractal formalism for selfsimilar functions under the action of nonlinear dynamical systems. Constr. Approx. 1994, 15, 209-240. [CrossRef]

31. Ben Slimane, M. Multifractal formalism for selfsimilar functions expanded in singular basis. Appl. Comput. Harmon. Anal. 2001, 11, 387-419. [CrossRef]

32. Jaffard, S. The multifractal nature of the Lévy processes. Probab. Theory Related Fields 1999, 114, $207-227$. [CrossRef]

33. Ben Slimane, M. Some functional equations revisited: The multifractal properties. Integral Transf. Spec. Funct. 2003, 14, 333-348. [CrossRef]

34. Jaffard, S. The spectrum of singularities of Riemann's function. Rev. Math. Iberoam. 1996, 12, 441-460. [CrossRef]

35. Jaffard, S. On the Frisch-Parisi conjecture. J. Math. Pures Appl. 2000, 79, 525-552. [CrossRef]

36. Fraysse, A. Generic validity of the multifractal formalism. SIAM J. Math. Anal. Soc. Ind. Appl. Math. 2007, 39, 593-607. [CrossRef]

37. Fraysse, A.; Jaffard, S. How smooth is almost every function in Sobolev space? Rev. Math. Iberoam. 2006, 22, 663-682. [CrossRef]

38. Kestener, P.; Arneodo, A. Generalizing the wavelet-based multifractal formalism to vector-valued random fields: Application to turbulent velocity and vorticity 3D numerical data. Phys. Rev. Lett. 2004, 93, 044501. [CrossRef] [PubMed]

39. Abry, P.; Clausel, M.; Jaffard, S.; Roux, S.G.; Vedel, B. Hyperbolic wavelet transform: An efficient tool for multifractal analysis of anisotropic textures. Rev. Math. Iberoam. 2015, 31, 313-348. [CrossRef]

40. Abry, P.; Roux, S.-G.; Wendt, H.; Messier, P.; Klein, A.-G.; Tremblay, N.; Borgnat, P.; Jaffard, S.; Vedel, B.; Coddington, J.; et al. Multiscale Anisotropic Texture Analysis and Classification of Photographic Prints: Art scholarship meets image processing algorithms. IEEE Signal Proc. Mag. 2015, 32, 18-27. [CrossRef]

41. Arneodo, A.; Audit, B.; Decoster, N.; Muzy, J.-F.; Vaillant, C. Wavelet-based multifractal formalism: Applications to DNA sequences, satellite images of the cloud structure and stock market data. In The Science of Disasters; Bunde, A., Kropp, J., Schellnhuber, H.J., Eds.; Springer: Berlin/Heidelberg, Germany, 2002; pp. 27-102.

42. Aubry, J.-M.; Maman, D.; Seuret, S. Local behavior of traces of Besov functions: Prevalent results. J. Funct. Anal. 2013, 264, 631-660. [CrossRef]

43. Ben Braiek, H.; Ben Slimane, M. Directional regularity criteria. C. R. Acad. Sci. Paris Sér. I Math. 2011, 349, 385-389. [CrossRef]

44. Ben Slimane, M.; Ben Braiek, H. Directional and anisotropic regularity and irregularity criteria in Triebel wavelet bases. J. Fourier Anal. Appl. 2012, 18, 893-914. [CrossRef]

45. Ben Slimane, M. Wavelet characterizations of multi-directional regularity. Fractals 2012, 20, $245-256$. [CrossRef]

46. Clausel, M.; Vedel, B. Explicit constructions of operator scaling Gaussian fields. Fractals 2011, 19, $101-111$. [CrossRef]

47. Davies, S.; Hall, P. Fractal analysis of surface roughness by using spatial data. J. R. Stat. Soc. Ser. B Stat. Methodol. 1999, 61, 3-37. [CrossRef]

48. Jaffard, S. Pointwise and directional regularity of nonharmonic Fourier series. Appl. Comput. Harmon. Anal. 2010, 28, 251-266. [CrossRef]

49. Ponson, L.; Bonamy, D.; Auradou, H.; Mourot, G.; Morel, S.; Bouchaud, E.; Guillot, C.; Hulin, J.P. Anisotropic self-affine properties of experimental fracture surfaces. Int. J. Fracture 2006, 140, 27-37. [CrossRef]

50. Roux, S.-G.; Clausel, M.; Vedel, B.; Jaffard, S.; Abry, P. Self-Similar Anisotropic Texture Analysis: The Hyperbolic Wavelet Transform Contribution. IEEE Trans. Image Proc. 2013, 22, 4353-4363. [CrossRef] [PubMed] 
51. Sampo, J.; Sumetkijakan, S. Estimations of Hölder Regularities and Direction of Singularity by Hart Smith and Curvelet Transforms. J. Fourier Anal. Appl. 2009, 15, 58-79. [CrossRef]

52. Triebel, H. Interpolation Theory, Function Spaces, Differential Operators; North-Holland: Amsterdam, The Netherlands, 1978.

53. Aimar, H.; Gomez, I. Parabolic Besov regularity for the heat equation. Constr. Approx. 2012, 36, 145-159. [CrossRef]

54. Biermé, H.; Meerschaert, M.M.; Scheffler, H.-P. Operator scaling stable random fields. Stoch. Proc. Appl. 2007, 117, 312-332. [CrossRef]

55. Bonami, A.; Estrade, A. Anisotropic analysis of some Gaussian models. J. Fourier Anal. Appl. 2003, 9, $215-236$. [CrossRef]

56. Khalil, A.; Joncas, G.; Nekka, F.; Kestener, P.; Arneodo, A. Morphological Analysis of H I Features. II. Wavelet-based multifractal formalism. Astrophys. J. Suppl. Ser. 2006, 165, 512-550. [CrossRef]

57. Ben Mabrouk, A. An adapted group dilation anisotropic multifractal formalism for functions. J. Nonlinear Math. Phys. 2008, 15, 1-23. [CrossRef]

58. Ben Slimane, M.; Ben Abid, M.; Ben Omrane, I.; Halouani, B. Criteria of pointwise and uniform directional Lipschitz regularities on tensor products of Schauder functions. J. Math. Anal. Appl. 2018, 460, 496-515. [CrossRef]

59. Ben Slimane, M.; Ben Braiek, H. Baire generic results for the anisotropic multifractal formalism. Rev. Mater. Complut. 2016, 29, 127-167. [CrossRef]

60. Bownik, M. Atomic and molecular decomposition of anisotropic Besov spaces. Math. Z. 2005, 250, 539-571. [CrossRef]

61. Bownik, M.; Ho, K.-P. Atomic and molecular decomposition of anisotropic Triebel- Lizorkin spaces. Trans. Am. Math. Soc. 2005, 385, 1469-1510.

62. Farkas, W. Atomic and subatomic decompositions in anisotropic function spaces. Math. Nachr. 2000, 209, 83-113. [CrossRef]

63. Führ, H. Vanishing moment conditions for wavelet atoms in higher dimensions. Adv. Comput. Math. 2016, 42, 127-153. [CrossRef]

64. Garrigós, G.; Tabacco, A. Wavelet decompositions of anisotropic Besov spaces. Math. Nachr. 2002, 239, 80-102. [CrossRef]

65. Garrigós, G.; Hochmuth, R.; Tabacco, A. Wavelet characterizations for anisotropic Besov spaces with $0<p<1$. Proc. Edinb. Math. Soc. 2004, 47, 573-595.

66. Hochmuth, R. Wavelet characterizations for anisotropic Besov spaces. Appl. Comput. Harmon. Anal. 2002, 12, 179-208. [CrossRef]

67. Kamont, A. On the fractional anisotropic Wiener field. Probab. Math. Statist. 1996, 16, 85-98.

68. Rosiene, C.-P.; Nguyen, T.-Q. Tensor-product wavelet vs. Mallat decomposition: A comparative analysis. In Proceedings of the 1999 IEEE International Symposium on Circuits and Systems VLSI (Cat. No.99CH36349), Orlando, FL, USA, 30 May-2 June 1999; Volume 3, p. 431434.

69. Triebel, H. Theory of Function Spaces III; Monographs in Mathematics, 78; Birkhäuser: Basel, Switzerland, 2006.

70. Triebel, H. Wavelet Bases in Anisotropic Function Spaces. Funct. Space Differ. Oper. Nonlinear Anal. 2004, 370-387.

71. Berkolako, M.Z.; Novikov, I.-Y. Wavelet bases in spaces of differentiable functions of anisotropic smoothness. (Russian). Dokl. Akad. Nauk. 1992, 324, 615-618.

72. Berkolako, M.Z.; Novikov, I.-Y. Unconditional bases in spaces of functions of anisotropic smoothness. (Russian). Trudy Mat. Inst. Steklov. Issled. Teor. Differ. Funktsii Mnogikh Peremen. Prilozh. 1993, 204, 35-51.

73. DeVore, R.-A.; Konyagin, S.-V.; Temlyakov, V.-N. Hyperbolic wavelet approximation. Constr. Approx. 1998, 14, 1-26. [CrossRef]

74. Westerink, P.-H. Subband Coding of Images. Ph.D. Thesis, Delft University of Technology, Delft, The Netherlands, 1989.

75. Yu, T.-P.; Stoschek, A.; Donoho, D.-L. Translation and direction invariant denoising of 2D and 3D images: Experience and algorithms. Proc. SPIE 1996, 2825, 608619.

76. Zavadsky, V. Image approximation by rectangular wavelet transform. J. Math. Imaging Vis. 2007, 27, 129-138. [CrossRef] 
77. Pesquet-Popesu, B.; Lévy-Véhel, J.; Stochastic Fractal Models for Image Processing. IEEE Signal Proces. Mag. 2002, 19, 48-62. [CrossRef]

78. Ayache, A.; Léger, S.; Pontier, M. Drap brownien fractionnaire. Potential Anal. 2002, 17, 31-43. [CrossRef]

79. Lakhonchai, P.; Sampo, J.; Sumetkijakan, S. Shearlet transforms and Hölder regularities. Int. J. Wavelets Multiresolut. Inform. Proc. 2010, 8, 743-771. [CrossRef]

80. Nualtong, K.; Sumetkijakan, S. Analysis of Hölder regularities by wavelet-like transforms with parabolic scaling. Thai J. Math. 2005, 3, 275-283.

81. Donoho, D. Wedgelets: Nearly minimax estimation of edges. Ann. Stat. 1999, 27, 353-382. [CrossRef]

82. Guo, K.; Labate, D. Analysis and detection of surface discontinuities using the 3D continuous shearlet transform. Appl. Comp. Harm. Anal. 2011, 30, 231-242. [CrossRef]

83. Candès, E.; Donoho, D. Ridgelets: A key to higher-dimensional intermittency? Philos. Trans. R. Soc. Lond. Ser. A Math. Phys. Eng. Sci. 1999, 357, 2495-2509. [CrossRef]

84. Mallat, S. Challenges for the 21st century. In Applied Mathematics Meets Signal Processing, In Proceedings of the the International Conference on Fundamental Sciences: Mathematics and Theoretical Physics (ICFS 2000), Singapore, 13-17 March 2000; World Scientific: Singapore, 2001; pp. 138-161.

85. Fell, J.; Führ, H.; Voigtlaender, F. Resolution of the wavefront set using general continuous wavelet transforms. J. Fourier Anal. Appl. 2016, 22, 997-1058. [CrossRef]

86. Sun, G.; Leng, J.; Cattani, C. A framework for circular multilevel systems in the frequency domain. Symmetry 2018, 10, 101. [CrossRef]

87. Kamont, A. Isomorphism of some anisotropic Besov and sequence spaces. Studia Math. 1994, 110, 169-189. [CrossRef]

88. Lemarié, P.-G.; Meyer, Y. Ondelettes et bases hilbertiennes. Rev. Mat. Iberoam. 1986, 1, 1-8. [CrossRef]

89. Meyer, Y. Ondelettes et Opérateurs; Hermann: Paris, France, 1990.

90. Daubechies, I. Orthonormal bases of compactly supported wavelets. Commun. Pure Appl. Math. 1988, 41, 909-996. [CrossRef]

91. Rogers, C.A. Hausdorff Measures; Cambridge University Press: Cambridge, UK, 1970.

92. Jaffard, S.; Lashermes, B.; Abry, P. Wavelet Leaders in Multifractal Analysis. In Wavelet Analysis and Applications; Tao Q., Vai, M.I., Xu Y., Eds.; Applied and Numerical Harmonic Analysis; Birkhaüser Verlag: Basel, Switzerland, 2006; pp. 219-264.

93. Biferalea, L.; Procaccia, I. Anisotropy in turbulent flows and in turbulent transport. Phys. Rep. 2005, 414, 43-164. [CrossRef]

94. Hinze, J.O. Turbulence; McGraw-Hill: New York, NY, USA, 1975.

95. Greenspan, H.P. The Theory of Rotating Fluids; Cambridge University Press: Cambridge, UK, 1968.

96. Grujic, Z. Vortex stretching and anisotropic diffusion in the 3D Navier-Stokes equations. Contemp. Math. 2016, 666, 240-251.

97. Constantin, P. Geometric statistics in turbulence. SIAM Rev. 1994, 36, 73-98. [CrossRef]

98. Ran, Z. Statistical Theory of Isotropic Turbulence. Part IV: Multiscales and Cascade. Available online: https:/ / arxiv.org/pdf/1012.5151(accessed on 23 December 2010).

(C) 2019 by the authors. Licensee MDPI, Basel, Switzerland. This article is an open access article distributed under the terms and conditions of the Creative Commons Attribution (CC BY) license (http://creativecommons.org/licenses/by/4.0/). 